Rev. Int. Contam. Ambie. 36 (3) 755-774, 2020

https://doi.org/10.20937/RICA.53341

\title{
ESTADO DE LA VALORIZACIÓN DE BIORRESIDUOS DE ORIGEN RESIDENCIAL EN GRANDES CENTROS URBANOS
}

\author{
State of the valorization of household biowaste in large urban centers
}

\begin{abstract}
Verónica MANZI TARAPUÉS ${ }^{1,2 *}$, Laura Isabella RENDÓN MUÑOZ², Marisol HERRERA RODAS², Mario Andrés GANDINI AYERBE ${ }^{2}$ y Luis Fernando MARMOLEJO REBELLÓN ${ }^{1}$
\end{abstract}

\author{
${ }^{1}$ Universidad del Valle, sede Meléndez, calle 13 100-00, Cali, Valle del Cauca, Colombia C.P. 760032 \\ ${ }^{2}$ Universidad Autónoma de Occidente, calle 25 115-85, Cali, Valle del Cauca, Colombia C.P. 760031 \\ *Autora para correspondencia: vmanzi@uao.edu.co
}

(Recibido: julio 2018; aceptado: noviembre 2019)

Palabras clave: residuos orgánicos, sistemas de gestión, ciudades, Latinoamérica, compostaje, digestión anaerobia, aprovechamiento

\section{RESUMEN}

En este estudio se revisó el estado del arte de la valorización de biorresiduos de origen residencial (BOR) en grandes centros urbanos (GCU) de países en desarrollo de América Latina y el Caribe (ALC) y países de alto ingreso económico (AIE) a nivel mundial. A partir del análisis de la literatura científica y técnica de entidades oficiales de los dos contextos evaluados, se estudiaron 70 GCU (37 de ALC y 33 de países de AIE) de 34 países. Los resultados señalaron que la valorización de los BOR es una práctica establecida en al menos 27 GCU, tres de ellos en ALC y 24 en AIE de Norteamérica, Europa, Asia y Oceanía. En éstos se identificó la implementación de 14 sistemas-tipo. Aunque con diferencias entre los contextos evaluados, los métodos de tratamiento más usados son el compostaje y la digestión anaerobia. Otros métodos utilizados son el procesamiento térmico para producción de alimentos para animales y el tratamiento mecánico biológico (TMB) con compostaje y digestión anaerobia. Se evidenció que los sistemas implementados producen compost (empleado principalmente en la jardinería y la agricultura), energía eléctrica, calor, combustible vehicular y alimentos para animales. Por último, pese a la relevancia del flujo de BOR en los GCU de ALC y los beneficios de su valorización, los resultados de este estudio evidenciaron la predominancia de la disposición final de BOR en este contexto, para el cual se recomienda establecer a los BOR como una fracción prioritaria cuya valorización debe ser la finalidad de los sistemas de gestión.

Key words: organic waste, management systems, cities, Latin America, composting, anaerobic digestion, recovery

\begin{abstract}
In the current study a literature review of household biowaste valorization in large urban centers of developing countries in Latin America and the Caribbean, as well as high-income countries worldwide, was performed. From the analysis of scientific and technical literature of official entities of the two evaluated contexts, 70 large urban centers (37 from Latin America and the Caribbean and 33 from high-income countries), from a total of 34 countries, were studied. The results indicated that household
\end{abstract}


biowaste valorization is established in at least 27 large urban centers, three of them in Latin America and the Caribbean and 24 in high-income countries of North America, Europe, Asia and Oceania. In these, the implementation of 14 type-systems was identified. Although there are differences between the evaluated contexts, the most used treatment methods are composting and anaerobic digestion. Other methods used are thermal processing for animal food production and mechanical biological treatment with composting and with anaerobic digestion. It was evidenced that the implemented systems produce compost (mainly used in gardening and agriculture), electric power, heat, vehicular fuel and animal feed. Finally, despite the relevance of the household biowaste stream in Latin America and the Caribbean large urban centers, and the benefits of its recovery, the results of this study showed the predominance of household biowaste landfilling in this context. In consequence, it is recommended to establish the household biowaste as a priority fraction whose valorization should be the primary aim of management systems.

\section{INTRODUCCIÓN}

Con el $78 \%$ de la población de América Latina y el Caribe (ALC) habitando en zonas urbanas, la gestión de biorresiduos de origen residencial (BOR) en esta región es un problema complejo, especialmente en los grandes centros urbanos (GCU). Los BOR, conformados por residuos de alimentos (BOR-A) y de jardín (BOR-J) generados en los hogares, son una fracción significativa de los residuos sólidos municipales (RSM), toda vez que representan hasta dos terceras partes de los residuos sólidos residenciales en países en desarrollo (Coffey y Coad 2010), los cuales, a su vez, son el flujo predominante de los RSM a nivel de ALC (BID 2015) - en promedio el $67 \%$ (Tello et al. 2010) - y a nivel global (Kawai y Tasaki 2016). Asimismo, en conjunto con los residuos biodegradables procedentes de mercados, restauración colectiva, industrias de alimentos, poda de árboles y corte de césped de áreas públicas, los BOR forman parte de los biorresiduos municipales (CE 2015a), los cuales constituyen a nivel global el $44 \%$ de los RSM (Kaza et al. 2018). No obstante, por su origen residencial, que demanda un compromiso activo de los ciudadanos (EC 2016), los BOR podrían tener un alto potencial de contaminación, lo que incrementa los desafíos de su valorización.

Los sistemas de gestión de biorresiduos responden a una finalidad que, según las definiciones de la CE (2015a), puede ser de valorización o de eliminación. La valorización de biorresiduos, entendida como la recuperación de nutrientes (reciclaje) y de energía, es la base de la gestión integrada circular de esta fracción (Cobo et al. 2018). Además, la gestión de biorresiduos es considerada como la columna vertebral de los sistemas de gestión de residuos (MAAMA 2013), dado que su reciclaje no sólo posibilita la obtención de productos finales valiosos, sino que su gestión diferenciada conduce al incremento significativo de las tasas globales de reciclaje, mejores resultados en la recuperación de otras fracciones de materiales (MAAMA 2013, EEA 2016), reducción de impactos ambientales y alargamiento de la vida útil de los rellenos sanitarios (Vögeli et al. 2014).

Por lo tanto, la alta proporción de biorresiduos en los RSM de países en desarrollo (Hoornweg y Bhada-Tata 2012, Wilson et al. 2012, UNEP-ISWA 2015) sugiere la conveniencia de un enfoque de valorización, en lugar de la eliminación en sus GCU. Sin embargo, la más reciente información regional encontrada, emitida una década atrás, reveló una incipiente valorización de biorresiduos en ALC (Tello et al. 2010), además evidenció una necesidad de actualización.

Al respecto, el Banco Interamericano de Desarrollo relacionó algunas experiencias de compostaje en GCU de ALC (Tello et al. 2010). Si bien esta evaluación no aportó información técnica de los sistemas, del flujo de biorresiduos tratados (si incluye o no a los BOR), ni de su grado de implementación, sí concluyeron que las prácticas de compostaje no se encuentran proporcionalmente implementadas - según la relevancia de los biorresiduos- en los RSM generados en ALC (Tello et al. 2010).

Por su parte, en países de alto ingreso económico (AIE) se evidencia una mayor implementación de la valorización de biorresiduos (EEA 2013, USEPA 2016). No obstante, al igual que en los países en desarrollo, en éstos se presentan desafíos para su gestión (Levis et al. 2011, EEA 2013, NSWEPA 2014). Por ejemplo, mientras, en 2010 la Unión Europea envió a disposición final, en promedio, el $40 \%$ del total de los biorresiduos y hasta el $100 \%$ en algunos estados miembros (JRC-IES 2011), en 2014, los EUA 
eliminaron el $76.3 \%$ de residuos de alimentos y el $31.6 \%$ de residuos de jardín (USEPA 2016). En este sentido, la comprensión de los aspectos técnicos que han determinado el desvío de los biorresiduos de rellenos sanitarios, y más aún su valorización, son de interés para autoridades locales, nacionales y regionales de países alrededor del mundo.

La literatura revisada evidenció que, a la fecha, se han publicado los resultados de evaluaciones regionales (OPS 2005, Tello et al. 2010, EEA 2013, BID 2015, EEA 2016) y nacionales (USEPA 2016) del estado de la gestión de RSM en diferentes contextos, aspectos que influyen en el reciclaje de RSM (23 países) (Troschinetz y Mihelcic 2009) y el estado de la gestión de residuos de alimentos en países en desarrollo (Thi et al. 2015). Otros estudios se han enfocado al análisis comparativo de la gestión de RSM en distintas ciudades alrededor del mundo. Entre ellos, los estudios realizados por UN-Habitat (2010) y Wilson et al. (2012) en 20 ciudades de países de alto, medio y bajo ingreso económico; 11 de ellas GCU (dos de ALC); asimismo, el análisis realizado por Abarca et al. (2013) sobre los factores que influyen en los sistemas de gestión de residuos en 36 ciudades de países en desarrollo a escala mundial.

Aun cuando estos estudios han evaluado aspectos técnicos o de gobernanza de la gestión de los RSM, presentando información y resultados consolidados a nivel de países y ciudades sobre la gestión de residuos, se han enfocado, en menor medida, a los biorresiduos (o alguna de sus fracciones), analizando principalmente el elemento de tratamiento. En la revisión realizada no se encontraron estudios enfocados al análisis de sistemas de gestión de BOR, con fines de valorización, que consideraran sus elementos y su interrelación.

De acuerdo con esto, la valorización de los BOR supone un desafío para la gestión de los RSM en todos los contextos. No obstante, la poca documentación de los sistemas en funcionamiento a nivel mundial contrasta con los altos requerimientos técnicos para su implementación en GCU. Por lo tanto, el análisis de las experiencias de valorización de BOR es útil para apoyar procesos de toma de decisiones para la gestión de esta fracción de residuos, especialmente en GCU de países en desarrollo. El objetivo de este estudio fue analizar los aspectos técnicos y la interrelación de los elementos que constituyen los sistemas de valorización de BOR en funcionamiento en GCU alrededor del mundo, e identificar los sistemas-tipo implementados y las similitudes y contrastes entre los contextos evaluados; también, proveer consideraciones futuras en el contexto de países en desarrollo en la región de ALC.

\section{MATERIALES Y MÉTODOS}

\section{Selección de literatura}

Para garantizar una base científica y técnica confiable y actualizada se seleccionaron fuentes de información de alta calidad y recientes. Éstas se conformaron por artículos científicos; documentos técnicos y de planificación nacionales y locales, disponibles en bases de datos como ScienceDirect, Academic Search Premiere y Scopus; y páginas de internet oficiales de las entidades gubernamentales y no gubernamentales de las regiones, países y ciudades estudiadas, los cuales fueron publicados en el periodo comprendido entre 2010 y 2017, en idiomas como español, inglés, portugués, francés, alemán, catalán y danés.

\section{Selección de GCU referentes de valorización de BOR}

Inicialmente se definió un grupo de GCU objeto de estudio conformado por 70 ciudades de 34 países en cinco regiones de dos contextos (países en desarrollo de ALC y países de AIE) (Cuadro I). En ALC, fueron objeto de estudio $37 \mathrm{GCU}$, ya que la información disponible limitó la consideración del total; entre estos, se encuentra el caso de Ciudad Juárez, León, Zapopan, Heroica Puebla, Ciudad Nezahualcóyotl, Maracaibo, Córdoba, Guayaquil y La Habana. Por su parte, en el grupo de países de AIE se estudiaron 33 GCU.

En segunda instancia, entre los GCU objeto de estudio, se seleccionó un grupo de GCU referentes, con experiencias significativas y representativas de valorización de BOR, conforme a los siguientes criterios: $i$ ) en el sistema de gestión implementado en el GCU, los materiales o energía obtenidos sustituyen a aquellos generados por otras fuentes, como resultado principal (según lo definido por la CE [2015a]), por lo que el producto final es utilizado; $i i$ ) cuenta con información técnica que permite su caracterización, y iii) considerando los mayores avances en la gestión de biorresiduos en el contexto de países AIE, en éste se seleccionaron los GCU que han implementado sistemas de valorización de BOR a nivel de ciudad, mientras que en el caso de países de ALC - a fin de localizar GCU referentes que apuestan por un enfoque de valorización-, se seleccionaron todas las experiencias de valorización, sin importar su grado de implementación. Por consiguiente, los GCU de AIE para los cuales se reportaron experiencias de valorización de BOR en etapas tempranas de implementación (por ejemplo, a nivel de prueba piloto) no fueron considerados. 
CUADRO I. GRUPO DE GRANDES CENTROS URBANOS OBJETO DE ESTUDIO

\begin{tabular}{|c|c|c|c|c|c|}
\hline Región & Contexto & País & Núm. & GCU objeto de estudio & Núm. \\
\hline ALC & En desarrollo & $\begin{array}{l}\text { México, Brasil, Colombia }{ }^{1}, \\
\text { Argentina, Ecuador, Bolivia, } \\
\text { Uruguay, Costa Rica, Venezuela, } \\
\text { República Dominicana, } \\
\text { Perú, Chile }^{1}\end{array}$ & 12 & $\begin{array}{l}\text { Ciudad de México, Ecatepec de Morelos, Mexicali, Gua- } \\
\text { dalajara, Monterrey, Tijuana, São Paulo, Rio de Janeiro, } \\
\text { Porto Alegre, Campinas, Goania, São Luís, Guarulhos, } \\
\text { Recife, Maceió, Belém, São Gonçalo, Fortaleza, } \\
\text { Belo Horizonte, Salvador, Curitiba, Manaus, Brasília, } \\
\text { Bogotá, Medellín, Cali, Barranquilla, Buenos Aires, Ro- } \\
\text { sario, Lima, Quito, Santa Cruz de la Sierra, Montevideo, } \\
\text { San José, Caracas, Santo Domingo, Santiago de Chile }\end{array}$ & 37 \\
\hline \multicolumn{2}{|c|}{ NorteaméricaAIE ${ }^{2}$} & Canadá, Estados Unidos & 2 & $\begin{array}{l}\text { Ottawa, Toronto, Vancouver, San Francisco, Nueva } \\
\text { York, San Antonio }\end{array}$ & 6 \\
\hline Europa & $\mathrm{AIE}^{2}$ & $\begin{array}{l}\text { Austria, Alemania, Irlanda, Bél- } \\
\text { gica, Dinamarca, Eslovaquia, Es- } \\
\text { lovenia, España, Francia, Grecia, } \\
\text { Italia, Noruega, Países Bajos, Por- } \\
\text { tugal, Reino Unido, Suecia, Suiza }\end{array}$ & 17 & $\begin{array}{l}\text { Viena, Berlín, Múnich, Dublín, Bruselas, Copenhague, } \\
\text { Bratislava, Liubliana, Madrid, Barcelona, Mallorca, } \\
\text { Principado de Asturias, París, Atenas, Milán, Oslo, } \\
\text { Ámsterdam, Lisboa, Londres, Estocolmo, Zúrich }\end{array}$ & 21 \\
\hline Asia & $\mathrm{AIE}^{2}$ & República de Corea, Japón & 2 & Seúl, Daejeon, Tokio, Chiba & 4 \\
\hline Oceanía & $\mathrm{AIE}^{2}$ & Australia & 1 & Sídney, Adelaida & 2 \\
\hline \multicolumn{3}{|c|}{ Total de países considerados } & 34 & Total de GCU estudiados & 70 \\
\hline
\end{tabular}

${ }^{1}$ Pertenece a la Organización para la Cooperación y el Desarrollo Económicos (UN 2014). ${ }^{2}$ excepto por República de Corea, los países de AIE responden a la clasificación de economías desarrolladas de acuerdo con UN (2014). AIE: alto ingreso económico. ALC: América Latina y El Caribe. GCU: gran centro urbano

Cabe señalar que en este estudio se consideraron como GCU ciudades con una población superior a un millón de habitantes en su zona urbana y otras ciudades principales, como las capitales de países de AIE que no alcanzaban esta cifra en 2013. Además, los países de AIE a los que pertenecen las ciudades estudiadas responden a la clasificación establecida por el Banco Mundial (es decir, tienen un ingreso nacional bruto per cápita mayor a \$12615 USD) (UN 2014) y son miembros activos de la Organización de Cooperación para el Desarrollo Económico (OCDE) (Cuadro I).

\section{Análisis de los sistemas de valorización de BOR de los GCU referentes e identificación de sistemas- tipo}

Se caracterizaron los sistemas de gestión implementados para la valorización de BOR, reconociendo los aspectos técnicos de los siguientes elementos: $i$ ) separación en la fuente (flujos de los residuos que se gestionan), $i$ i) presentación (tipos de contenedores), iii) recolección, $i v$ ) tratamiento o disposición final y v) productos finales y uso.

Considerando que los procesos en funcionamiento en los GCU referentes se podrían relacionar con una tendencia en la implementación de sistemas de valorización de BOR alrededor del mundo, se identificó un conjunto de sistemas-tipo en los contextos estudiados, los cuales corresponden a combinaciones de esquemas y métodos de los diferentes elementos que los componen. Así, una vez caracterizados, los sistemas de gestión implementados en los GCU referentes fueron clasificados de acuerdo con características similares en todos los elementos, conformando los sistemas-tipo. En el elemento de tratamiento, la clasificación se realizó según el método de tratamiento principal, en caso de contar con postratamientos (por ejemplo, digestión anaerobia con compostaje del digestato), o según el método de tratamiento de BOR-A, cuando se lleva a cabo la gestión diferenciada de las fracciones de BOR.

\section{RESULTADOS Y DISCUSIÓN}

\section{Estado de la valorización de BOR en los GCU de países en desarrollo de ALC}

Los BOR son una fracción de interés en el flujo de residuos de los GCU de ALC. Estudios 
de caracterización realizados en algunas ciudades revelan que su proporción es del $47 \%$ al $80 \%$ de los residuos sólidos residenciales. Es el caso de ciudades como Quito, con el $53.94 \%$ (Castillo 2012); Buenos Aires, con el $47.48 \%$ (FIUBA-CEAMSE 2011); Mexicali, con el $80 \%$ (Ojeda-Benítez et al. 2003); Bogotá, con el 61.43 \% (UAESP 2011);
Medellín, con el 55,39\%(UDEM 2015), y Cali, con el $65.54 \%$ (DAPM-Univalle 2006).

Pese a la relevancia de los BOR, entre los 37 GCU estudiados en ALC, sólo se pudo evidenciar la valorización de esta fracción en tres de ellos, considerados en este estudio como GCU referentes de este contexto (Cuadro II). En las ciudades

\section{CUADRO II. ENFOQUE DE GESTIÓN DE BIORRESIDUOS DE ORIGEN RESIDENCIAL EN LOS GRANDES CENTROS URBANOS OBJETO DE ESTUDIO DE AMÉRICA LATINA Y EL CARIBE, Y SELECCIÓN DE GRANDES CENTROS URBANOS REFERENTES DE VALORIZACIÓN}

\begin{tabular}{|c|c|c|c|c|c|}
\hline \multirow{2}{*}{$\begin{array}{l}\text { Población } \\
\text { urbana } \\
\text { (millones de } \\
\text { habitantes) }\end{array}$} & \multirow{2}{*}{$\begin{array}{l}\text { Núm. de } \\
\text { GCU objeto } \\
\text { de estudio }\end{array}$} & \multicolumn{3}{|c|}{ Enfoque de gestión de BOR de los GCU objeto de estudio } & \multirow[t]{2}{*}{ Fuentes de información } \\
\hline & & $\begin{array}{l}\text { Valorización } \\
\text { (GCU referente) }\end{array}$ & Eliminación(GCU co & n disposición final y otros) & \\
\hline $1-2$ & 21 & Rosario $^{\mathrm{a}, \mathrm{b}}$ & $\begin{array}{l}\text {-Ecatepec de } \text { Morelos }^{\mathrm{c}} \\
\text {-Mexicali } \\
\text {-Guadalajara }^{\mathrm{e}} \\
\text {-Monterrey } \\
\text {-Tijuana }^{\mathrm{g}} \\
\text {-Campinas } \\
\text {-Goiânia }^{\mathrm{h}} \text { Recife }^{\mathrm{j}} \\
\text {-Maceiók }{ }^{\mathrm{k}} \text { Belém }^{\mathrm{i}} \\
\text {-São Gonçalo }\end{array}$ & $\begin{array}{l}\text {-São Luís }{ }^{\mathrm{i}} \\
\text {-Guarulhos }^{1,1} \\
\text {-Quito } \\
\text {-Santa Cruz de la Sierra }^{\mathrm{n}, \tilde{n}} \\
\text {-Porto Alegre }^{\mathrm{o}, 2} \\
\text {-Montevideo }^{\mathrm{p}} \\
\text {-San Joséq }^{\mathrm{q}} \\
\text {-Caracas }^{\mathrm{r}} \\
\text {-Barranquilla } \\
\text {-B,t }\end{array}$ & 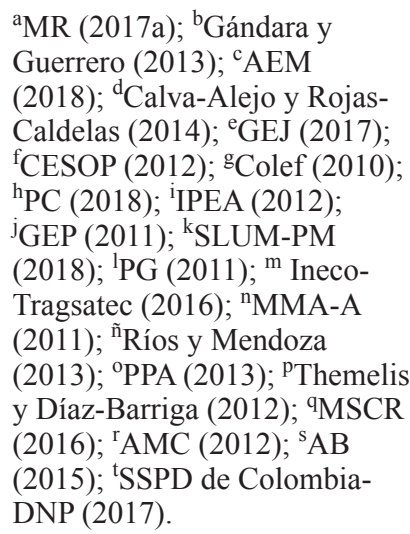 \\
\hline $2-5$ & 11 & Medellín $n^{\mathrm{a}, \mathrm{b}}$ & $\begin{array}{l}\text {-Brasilia }{ }^{\mathrm{c}, 3} \\
\text {-Fortaleza } \\
\text {-Belo Horizonte } \\
\text {-Salvador } \\
\text {-Curitiba } \\
\text { - }\end{array}$ & $\begin{array}{l}\text {-Manaus }^{\mathrm{i}} \\
\text {-Buenos Aires }^{\mathrm{j}, \mathrm{k}, 3} \\
\text {-Cali }^{\mathrm{l}, \mathrm{b}} \\
\text {-Santo Domingo } \\
\text {-Santiago de } \\
\text {-Chile }^{\mathrm{n}}\end{array}$ & 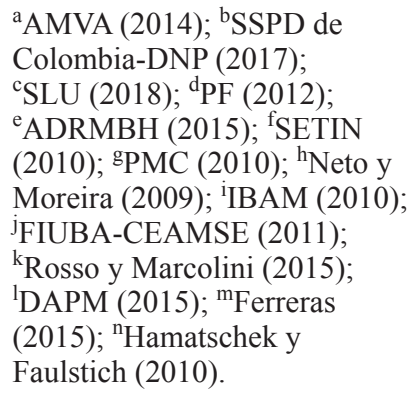 \\
\hline$>5$ & 5 & $\begin{array}{l}\text { Ciudad de } \\
\text { México }^{\text {a }}\end{array}$ & $\begin{array}{l}\text {-São Paulo } \\
\text {-Río de } \\
\text {-Janeiro } \\
\text { c,d,e }\end{array}$ & $\begin{array}{l}- \text { Lima }^{\mathrm{f}} \\
- \text { Bogotá }^{\mathrm{g}, \mathrm{h}}\end{array}$ & 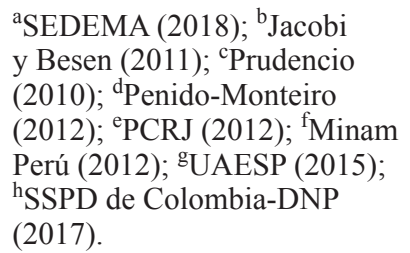 \\
\hline
\end{tabular}

\begin{tabular}{llll}
\hline Total GCU & 37 & 3 & 34
\end{tabular}

${ }^{1}$ Se reporta un proyecto de compostaje de BOR en el huerto municipal; no obstante, la información disponible es insuficiente para su caracterización. ${ }^{2}$ Hay una planta de clasificación y compostaje de RSM que en 2011 trató 10.7 t/d de BOR; sin embargo, se documenta que los BOR tratados provienen de la recolección conjunta y no se evidencia su valorización. ${ }^{3}$ Se reporta TMB con compostaje: planta Bella Vista, en Buenos Aires, con capacidad instalada de 1100 t/d de RSM, y plantas Ceilandia y Asa Sul en Brasília-Ceilandia, con capacidades instaladas aproximadas de 600 y 300 t/d, respectivamente (Mansur 2015, GB 2018); GCU: gran centro urbano 
restantes se encontró que la gestión de BOR se realiza con un enfoque de eliminación, donde esta fracción es parte de flujos residuales destinados a la disposición final (Cuadro II). Según las características e interrelación de los elementos de los sistemas implementados, en estos GCU referentes se reconoce la implementación de tres sistemas-tipo para la valorización de BOR (Cuadro III), que si bien tienen en común el tipo de presentación (en bolsas plásticas), el producto final y el uso (compost/aplicado al suelo), difieren en los esquemas de separación en la fuente, tipo de recolección, método de tratamiento y tipo de instalación (centralizado o descentralizado) (Cuadro III).

El sistema-tipo1 representa la situación de Rosario, en la que el flujo de residuos provenientes de la recolección domiciliaria, que contiene a los BOR, se procesa en una instalación de tratamiento mecánico biológico (TMB), donde se realiza una clasificación manual y mecánica para la recuperación de reciclables secos, seguida por un proceso de compostaje. En este sistema, el uso del producto final (Cuadro III) se realiza con previa confirmación de su calidad (tanto fisicoquímica como microbiológica) (MR 2017a).

El sistema-tipo 2 representa la experiencia de Medellín. La capacidad instalada de este sistema revela un bajo nivel de implementación (Cuadro III) en este, el conjunto de los BOR (mezcla de BOR-A y BOR-J) separados en la fuente y recogidos selectivamente por recicladores organizados, es tratado mediante compostaje (descentralizado a pequeña escala). De este modo, en la zona urbana existen 92 instalaciones, 55 de las cuales tratan los BOR generados en 38 condominios residenciales/multiusuarios (Acodal-AMVA 2013), en el marco del proyecto de Eco-Huertas (SGCT-UDEM 2015).

Por su parte, el sistema-tipo 3, implementado en Ciudad de México, representa la experiencia más significativa de ALC debido a la capacidad de tratamiento instalada (Cuadro III), con Bordo Poniente como la planta de compostaje de biorresiduos separados en la fuente documentada más grande de la región, que de acuerdo con la Secretaría del Medio Ambiente de la ciudad es de 2500 t/d (SEDEMA 2018). El compost producido es apto para su uso en áreas verdes y agricultura (tipo B, según SMADF [2012]) gracias, en gran medida, a la aplicación de la norma que estableció criterios y especificaciones técnicas de separación en la fuente, clasificación, recolección selectiva y almacenamiento de residuos (SMADF 2015).

En comparación con Medellín y Ciudad de México, Rosario ha implementado una gestión no diferenciada. Sin embargo, Rosario reporta la valorización de residuos (incluidos los BOR) como finalidad del sistema implementado (MR 2017a), así como la ejecución de una prueba piloto de separación en

CUADRO III. CARACTERÍSTICAS DE LOS SISTEMAS DE VALORIZACIÓN DE BIORRESIDUOS DE ORIGEN RESIDENCIAL EN LOS GRANDES CENTROS URBANOS REFERENTES DE AMÉRICA LATINA Y EL CARIBE

\begin{tabular}{lllllll}
\hline $\begin{array}{l}\text { Sistema- } \\
\text { tipo }\end{array}$ & SF/presentación & $\begin{array}{l}\text { Tipo de } \\
\text { recolección }\end{array}$ & $\begin{array}{l}\text { Método de } \\
\text { tratamiento }\end{array}$ & $\begin{array}{l}\text { Producto final/ } \\
\text { uso }\end{array}$ & $\begin{array}{l}\text { GCU (generación } \\
\text { de BOR en t/d) }\end{array}$ & Fuentes de información \\
\hline 1 & $\begin{array}{l}\text { Flujo de residuos } \\
\text { mezclados/bolsas } \\
\text { plásticas }\end{array}$ & Conjunta & $\begin{array}{l}\text { TMB con } \\
\text { compostaje }\end{array}$ & $\begin{array}{l}\text { Compost/aplica- } \\
\text { ción en parques y } \\
\text { áreas públicas } 1\end{array}$ & Rosario (ND) & MR (2017a) \\
\hline $\begin{array}{l}\text { BOR } / \text { bolsas } \\
\text { plásticas }\end{array}$ & $\begin{array}{l}\text { Selectiva } \\
\text { (manual) }\end{array}$ & $\begin{array}{l}\text { Compostaje } \\
\text { comunitario }\end{array}$ & $\begin{array}{l}\text { Compost/aplica- } \\
\text { ción en el suelo }\end{array}$ & Medellín $\left(680^{3}\right)$ & $\begin{array}{l}\text { AMVA (2014), Acodal- } \\
\text { AMVA (2013), SSPD de } \\
\text { Colombia-DNP (2017), } \\
\text { UDEM (2015) }\end{array}$ \\
\hline 3 & BOR $/$ bolsas & Selectiva & Compostaje & $\begin{array}{l}\text { Compost/aplica- } \\
\text { ción en cultivos }\end{array}$ & $\begin{array}{l}\text { Ciudad de México } \\
\left(2928^{3}\right)\end{array}$ & SEDEMA (2015, 2018) \\
\hline
\end{tabular}

Tecnologías identificadas (GCU, núm. de plantas, capacidad instalada en $\mathrm{t} / \mathrm{d}$ )

ND (Rosario, 1 planta, 2004), compostaje modular (Medellín, 92 plantas, 91.9 ${ }^{5}$ ), pilas estáticas con volteo mecánico (Ciudad de México, 8 plantas, 25316)

SF: separación en la fuente, GCU: gran centro urbano, BOR: biorresiduos de origen residencial, TMB: tratamiento mecánico biológico, ND: no disponible. ${ }^{1}$ También se utiliza como material de cobertura del relleno sanitario. ${ }^{2}$ Se refiere al conjunto de los BOR, es decir, a la mezcla de BOR-A y BOR-J. ${ }^{3}$ Calculado a partir de información reportada en las fuentes consultadas. ${ }^{4}$ Se refiere al flujo de residuos mezclados. ${ }^{5} \mathrm{Se}$ refiere a BOR. ${ }^{6} \mathrm{Se}$ refiere a biorresiduos municipales, suma de capacidades de tratamiento de ocho plantas de compostaje en Ciudad de México (SEDEMA 2018) 
la fuente y recolección selectiva de BOR (MR $2017 b$ ). Esto sugiere que podría estar en una transición hacia una gestión diferenciada de BOR.

Por otro lado, el enfoque de descentralización adoptado por Medellín establece diferencias frente a los otros sistemas, tanto en términos de recolección como de tratamiento. Si bien su capacidad instalada no es comparable con la de Ciudad de México, es importante señalar que Medellín tiene proyectado implementar entre 10 a 12 plantas de compostaje de 40 a $45 \mathrm{t} / \mathrm{d}$ para aumentar la cobertura de la valorización de los BOR (SGCT-UDEM 2015). Este escenario podría sugerir que Medellín le apuesta a un esquema de gestión que concuerda con lo recomendado por Drescher y Zurbrügg (2006) para grandes ciudades de países en desarrollo: una combinación de plantas de pequeña escala descentralizadas con plantas centralizadas de tamaño medio.

Estas experiencias identificadas en Rosario, Medellín y Ciudad de México indican un interés de ALC por el aprovechamiento de los BOR. Sin embargo, también ratifican que, al igual que para los residuos de alimentos en los países en desarrollo (Thi et al. 2015), en los GCU de ALC el destino principal de los BOR es la disposición final. Debido al grado de implementación de los sistemas en funcionamiento (Cuadro III), aun en estos GCU referentes - aunque en menor proporción en Ciudad de México-, una parte significativa de los BOR se envía a la disposición final, tal como sucede para el total de los BOR generados en la mayoría de los GCU objeto de estudio (Cuadro II). Esta situación revela pocos avances en comparación con lo reportado para ALC por Tello et al. (2010) y BID (2015) para el flujo de RSM en general (que contiene a los BOR).

La predominancia de la disposición final de BOR puede constituir un riesgo ambiental, toda vez que la vida útil de la infraestructura no es finita y, en una perspectiva de impactos de largo plazo, incluso los rellenos sanitarios tecnológicamente más sofisticados presentan fallas que materializan las emisiones potenciales aisladas mediante los mecanismos de control implementados (Christensen et al. 2011). Esta situación es más crítica en ALC, donde se ha reportado la existencia de rellenos sanitarios con inconvenientes de operación y control (Noguera y Olivero 2010), así como la persistencia de prácticas inadecuadas de disposición final (BID 2015).

Respecto a la disposición final, vale la pena mencionar que la información disponible no fue suficiente para clasificar las infraestructuras utilizadas. Pese a esto, las fuentes consultadas (Cuadro II) reportan como adecuados los sitios actuales de disposición final de 13 de los 37 GCU objeto de estudio: Ciudad de México (reportado como relleno sanitario autorizado), São Paulo y Belo Horizonte (reportados como rellenos regularizados), Guarulhos, Rosario, Bogotá, Medellín, Cali, Barranquilla, Buenos Aires, San José, Quito y Lima (reportados como rellenos sanitarios).

Finalmente, en algunos GCU de ALC también se identificaron sistemas de gestión no diferenciada de BOR que no constituyen valorización, como el sistema de TMB con compostaje en Buenos Aires (Cuadro II) que desvía los BOR de la disposición final, reportando el uso del material estabilizado como cobertura primaria del relleno sanitario. Éste es también el caso de Bogotá, Monterrey y Sao Paulo (Cuadro II), que recuperan energía del biogás generado en los rellenos sanitarios. Este biogás es considerado una de las mayores fuentes antrópicas de emisiones de metano (Kumar y Samadder 2017). Por lo tanto, su recuperación es una de las acciones de mitigación del cambio climático establecidas para el sector de residuos - como es el caso de Colombia (IDEAM 2017)—, aunque dicha recuperación se caracteriza por su ineficiencia (Kumar y Samadder 2017).

Estado de la valorización de BOR en países de AIE

Se analizó un grupo de 24 GCU referentes de valorización de BOR (Cuadro IV). Los nueve GCU restantes (Cuadro I) no cumplen con alguno de los criterios de selección establecidos. Es el caso de Nueva York (C40 Cities 2013), Atenas (SO 2015), Estocolmo (BiPro-CRI 2015) y el Principado de Asturias (COGERSA-GPA 2014), cuyos sistemas se reportaron en etapas tempranas de implementación; Chiba y Bratislava, para las cuales no se encontró información suficiente para su caracterización, y, por último, Ámsterdam (BiPro-CRI 2015, AEB Amsterdam 2018), Lisboa (BiPro-CRI 2015) y Tokio (CAT 23 Cities-SCICWM 2014), donde los BOR se gestionan en flujos de residuos mezclados mediante incineración. En éstos, la asociación entre energía recuperada y BOR tratados, que incluso podrían requerir energía para su secado, demanda un análisis fuera del alcance de este estudio (por ejemplo, de eficiencia energética [MAAMA 2013]), para su clasificación como sistemas de valorización energética de BOR.

A continuación se analizan los aspectos técnicos de los sistemas-tipo identificados en los GCU referentes de este contexto, a partir de la clasificación realizada por método de tratamiento (Cuadro IV) (compostaje, digestión anaerobia y otros). 
CUADRO IV. GRANDES CENTROS URBANOS REFERENTES DE VALORIZACIÓN DE BIORRESIDUOS DE ORIGEN RESIDENCIAL EN PAÍSES DE ALTO INGRESO ECONÓMICO POR TIPO DE TRATAMIENTO

\begin{tabular}{|c|c|c|c|c|}
\hline \multirow{2}{*}{$\begin{array}{l}\text { Población } \\
\text { (millones de } \\
\text { habitantes) }\end{array}$} & \multicolumn{4}{|c|}{ Grupos de GCU por método de tratamiento implementado } \\
\hline & Compostaje & Digestión anaerobia & $\begin{array}{l}\text { Producción de } \\
\text { alimentos para } \\
\text { animales }\end{array}$ & $\begin{array}{l}\text { TMB con digestión } \\
\text { anaerobia }\end{array}$ \\
\hline$<1$ & $\begin{array}{l}\text { Copenhague, Dublín, } \\
\text { Ottawa, San Francisco }\end{array}$ & $\begin{array}{l}\text { Liubliana, Mallorca, } \\
\text { Oslo, Vancouver, Zúrich. }\end{array}$ & - & - \\
\hline $1-2$ & Adelaida, San Antonio & $\begin{array}{l}\text { Barcelona, Bruselas, Milán, } \\
\text { Múnich, Viena. }\end{array}$ & Daejeon & - \\
\hline $2-5$ & París, Sídney & Berlín, Toronto. & - & Madrid \\
\hline$>5$ & Londres & - & Seúl & - \\
\hline $\begin{array}{l}\text { Número de } \\
\text { experiencias }\end{array}$ & 9 & 12 & 2 & 1 \\
\hline
\end{tabular}

GCU: gran centro urbano, TMB: tratamiento mecánico biológico

Sistemas de valorización de BOR en el grupo de GCU que han implementado el compostaje

En los GCU de este grupo, la valorización de los BOR se realiza mediante una gestión diferenciada. Se identificaron tres esquemas de separación en la fuente que definen los flujos gestionados: $i$ ) conjunto de BOR, ii) BOR-J, y iii) flujos independientes de BOR-A y BOR-J. Éstos se configuran, principalmente, por medio de sistemas (sistemas-tipo 4 a 8) en los cuales los BOR se presentan en contenedores retornables y su recolección es selectiva, y se valorizan mediante la producción de compost de alta calidad (Cuadro V).

Sistemas de valorización de BOR en el grupo de GCU que han implementado la digestión anaerobia

En este grupo, la valorización de los BOR también se realiza a través de sistemas de gestión diferenciada, en dos de los esquemas de separación en la fuente previamente mencionados: i) conjunto de BOR y ii) flujos independientes de BOR-A y BOR-J, y configuraciones del tratamiento que pueden incluir, además de la digestión anaerobia, el compostaje. Las características de los sistemas analizados condujeron al planteamiento de cuatro sistemas-tipo ( 9 a 12) en los cuales también predomina la presentación en contenedores retornables y la recolección selectiva, y se resalta la obtención de distintas formas de energía (Cuadro VI).
Sistemas de valorización de BOR en el grupo de GCU que han implementado otros métodos de tratamiento

En los GCU de este grupo se configuran dos sistemas-tipo (13 y 14). El primero está basado en el procesamiento térmico seco y húmedo de los BOR-A separados en la fuente, para la producción de alimentos de animales, como sucede en Seúl y Daejeon; el segundo, en la valorización energética de los BOR como parte de la fracción residual, mediante el uso del TMB con digestión anaerobia como tratamiento central. Este último es el caso de Madrid, donde también se reporta el uso de compost producido a partir de biorresiduos separados mecánicamente (Cuadro VII).

Sobre los sistemas de valorización de BOR en funcionamiento en GCU en países de ALC y AIE

Las experiencias de valorización de los GCU referentes alrededor del mundo se representan en 14 sistemas-tipo de valorización de BOR. En 12 de ellos, implementados en el $92 \%$ de los GCU referentes, se gestionan diferentes flujos de BOR separados en la fuente, en mayor medida mediante tratamientos biológicos, a partir de los cuales se obtienen compost y energía. En los sistemas tipo restantes (3 y 14), donde los BOR se gestionan mediante TMB, también se obtienen estos productos finales. No obstante, en esos sistemas el enfoque de gestión no diferenciada contrasta con la situación deseada; esto es, con la 
CUADRO V. CARACTERÍSTICAS DE LOS SISTEMAS DE VALORIZACIÓN DE BIORRESIDUOS DE ORIGEN RESIDENCIAL EN GRANDES CENTROS URBANOS DE PAÍSES DE ALTO INGRESO ECONÓMICO, MEDIANTE COMPOSTAJE

\begin{tabular}{|c|c|c|c|c|c|}
\hline Sistema-tipo & $\mathrm{SF} /$ presentación & $\begin{array}{l}\text { Tipo de } \\
\text { recolección }\end{array}$ & Producto final/uso & GCU & $\begin{array}{l}\text { Fuentes de } \\
\text { información }\end{array}$ \\
\hline 4 & $\begin{array}{l}\mathrm{BOR} / \\
\text { contenedor }\end{array}$ & Selectiva & $\begin{array}{l}\text { Compost/aplicación } \\
\text { en jardines, cultivos y } \\
\text { proyectos } \\
\text { paisajísticos }\end{array}$ & $\begin{array}{l}\text { - Dublín } \\
\text { - Ottawa, } \\
\text { - San Antonion } \\
\text { - San Anton } \\
\text { - San Francisco }{ }^{\mathrm{i}, \mathrm{j}, \mathrm{k}} \\
\text { - Adelaida } \\
\text { - A,l,m }\end{array}$ & $\begin{array}{l}{ }^{\mathrm{a} C r e ́ ~(2018) ; ~}{ }^{\mathrm{b}} \text { BiPro- } \\
\text { CRI (2015); }{ }^{\mathrm{E}} \mathrm{EPA} \\
(2016) ;{ }^{\mathrm{d}} \mathrm{VE}(2011) ; \\
{ }^{\mathrm{e}} \text { Orgaworld (2014); } \\
{ }^{\mathrm{f}} \mathrm{CO}(2016) ;{ }^{\mathrm{g}} \mathrm{CSA} \\
(2015) ;{ }^{\mathrm{h}} \mathrm{Hagney} \\
\text { (2016); }{ }^{\mathrm{i}} \mathrm{SFE}(2016) ; \\
{ }^{\mathrm{j}} \text { Recology }(2016) ; \\
{ }^{\mathrm{k}} \mathrm{SFE}(2017),{ }^{\mathrm{l} Z a m a n} \\
(2014) ;{ }^{\mathrm{m}} \mathrm{CA}(2019) .\end{array}$ \\
\hline 5 & $\begin{array}{l}\text { BOR-A crudos de } \\
\text { origen vegetal }^{1} \text { y } \\
\text { BOR-J/contenedor }\end{array}$ & Selectiva & $\begin{array}{l}\text { Compost/distribuido } \\
\text { gratuitamente a la } \\
\text { comunidad }\end{array}$ & Copenhague $^{\mathrm{a}, \mathrm{b}, \mathrm{c}}$ & $\begin{array}{l}{ }^{a} \mathrm{CC}(2014) ;{ }^{b} \text { Chang } \\
\text { y Pires }(2015) ;{ }^{c} \mathrm{KK} \\
(2014)\end{array}$ \\
\hline 6 & BOR/ND & No aplica $^{2}$ & $\begin{array}{l}\text { Compost/en jardines } \\
\text { públicos y privados }\end{array}$ & París $^{\mathrm{a}, \mathrm{b}, \mathrm{c}}$ & $\begin{array}{l}{ }^{a} \text { BiPro-CRI (2015); } \\
{ }^{b} \mathrm{MP}(2017) \text {; }{ }^{\mathrm{C}} \text { Paris } \\
\text { (2016). }\end{array}$ \\
\hline 7 & BOR-J/contenedor & Selectiva & $\begin{array}{l}\text { Compost/mejorador de } \\
\text { suelos en jardinería y } \\
\text { agricultura }\end{array}$ & Sidney ${ }^{4, a, b}$ & $\begin{array}{l}{ }^{\mathrm{a}} \mathrm{CS}(2011) ;{ }^{\mathrm{b}} \mathrm{CS} \\
(2017) .\end{array}$ \\
\hline \multirow[t]{3}{*}{8} & BOR/contenedor & \multirow[t]{3}{*}{ Selectiva } & \multirow{3}{*}{$\begin{array}{l}\text { Compost/mejorador del } \\
\text { suelo en cultivos locales }\end{array}$} & \multirow[t]{3}{*}{ Londres $^{5, \mathrm{a}, \mathrm{b}}$} & \multirow{3}{*}{$\begin{array}{l}{ }^{\mathrm{a}} \mathrm{CL}(2014) ;{ }^{\mathrm{b}} \mathrm{CL} \\
(2016) .\end{array}$} \\
\hline & BOR-A/contenedor & & & & \\
\hline & BOR-J/contenedor & & & & \\
\hline \multicolumn{2}{|c|}{$\begin{array}{l}\text { Tecnologías identificadas (GCU, } \\
\text { capacidad instalada en } \mathrm{t} / \mathrm{d} \text { ) }\end{array}$} & \multicolumn{4}{|c|}{$\begin{array}{l}\text { Hileras (San Antonio, ND), hileras cubiertas (San Francisco, 274), túnel (Ottawa, 220), } \\
\text { composteras comunitarias }^{6} \text { (París, ND) }\end{array}$} \\
\hline
\end{tabular}

SF: separación en la fuente, GCU: gran centro urbano, BOR: biorresiduos de origen residencial, BOR-A: fracción de residuos de alimentos en los BOR, BOR-J: fracción de residuos de jardín en BOR, ND: no disponible. ${ }^{1}$ Los BOR-A de origen animal se gestionan mezclados en la fracción resto, con recolección conjunta e incineración con recuperación de energía. ${ }^{2}$ No cuenta con sistema de recolección, ya que se lleva a cabo compostaje in situ: doméstico y comunitario. ${ }^{3} \mathrm{El}$ gobierno local ha estado promoviendo la gestión diferenciada de BOR-A, proporcionando contenedores y bolsas compostables para el almacenamiento temporal de residuos de alimentos en las cocinas de las residencias, para una presentación conjunta con los BOR-J. ${ }^{4}$ Los BOR-A se gestionan mezclados en la fracción resto; se tratan en una planta de TMB y el compost-como-salida obtenido es utilizado para rehabilitar suelos de zonas mineras. ${ }^{5}$ En 29 de los 33 distritos se realiza la recolección selectiva de BOR-J (en algunos de ellos en conjunto con BOR-A y en 17 de las fracciones independientes de BOR-A y BOR-J) (BiPro-CRI 2015); en algunos distritos, los flujos de BOR-A separados en la fuente son tratados mediante digestión anaerobia (RN 2018). ${ }^{6} 600 \mathrm{~L}$ de capacidad. También se reporta el uso de vermicompostaje en algunos casos

separación en la fuente como condición del reciclaje de biorresiduos con fines de obtención de productos finales para uso en suelos (MAAMA 2013, Saveyn y Eder 2014, Manfredi et al. 2015), que, para el caso de Colombia, es obligatoria (ICONTEC 2011). En este sentido, la falta de separación en la fuente podría restringir el uso del compost en estos sistemas.

Las características técnicas de los esquemas de separación en la fuente implementados revelan que, aunque en el $50 \%$ de los sistemas-tipo identificados en ALC y AIE se separa en la fuente el conjunto de BOR, en el contexto de AIE se han establecido otros esquemas de separación asociados con la gestión diferenciada de alguno o ambos flujos de las fracciones individuales de BOR. Es el caso del sistematipo 7, que solo valoriza los BOR-J (Cuadro V); de los sistemas-tipo 12 (Cuadro VI) y 13 (Cuadro VII), que valorizan únicamente los BOR-A, y del sistema-tipo 11, que, a diferencia de los demás, valoriza ambos flujos de manera independiente 
CUADRO VI. CARACTERÍSTICAS DE SISTEMAS DE VALORIZACIÓN DE BIORRESIDUOS DE ORIGEN RESIDENCIAL EN GRANDES CENTROS URBANOS DE PAÍSES DE ALTO INGRESO ECONÓMICO, MEDIANTE DIGESTIÓN ANAEROBIA

\begin{tabular}{|c|c|c|c|c|c|c|}
\hline \multirow{2}{*}{$\begin{array}{l}\text { Sistema- } \\
\text { tipo }\end{array}$} & \multirow{2}{*}{$\begin{array}{l}\mathrm{SF} / \\
\text { presentación }\end{array}$} & \multirow{2}{*}{$\begin{array}{l}\text { Tipo de } \\
\text { recolección }\end{array}$} & \multicolumn{2}{|c|}{ Método de tratamiento } & \multirow{2}{*}{ GCU } & \multirow{2}{*}{ Fuentes de información } \\
\hline & & & Métodos & Producto final/uso & & \\
\hline 9 & $\begin{array}{l}\text { BOR/ } \\
\text { contenedores }\end{array}$ & Selectiva & $\begin{array}{l}\text { Digestión } \\
\text { anaerobia }+ \\
\text { compostaje } \\
\text { del digestato }\end{array}$ & $\begin{array}{l}\text {-Biogás/energía } \\
\text { eléctrica y } \\
\text { calor }{ }^{1,3,4,5,6} \text {, } \\
\text { combustible para } \\
\text { instalaciones de } \\
\text { tratamiento }^{2} \text { y } \\
\text { vehículos de } \\
\text { recolección } \\
\text {-Digestato líquido/ } \\
\text { biofertilizante }^{4} \\
\text {-Compost/agricultura } \\
\text { y jardinería }{ }^{1,2,3,4,6}, \\
\text { como fertilizante } \\
\text { de alta calidad }\end{array}$ & 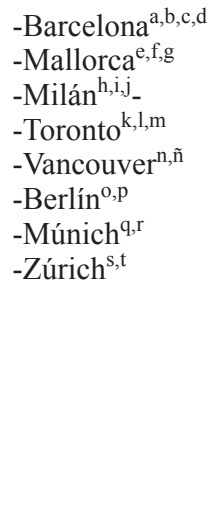 & 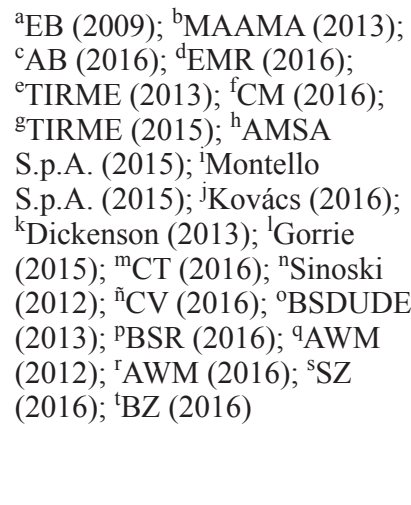 \\
\hline 10 & $\begin{array}{l}\text { BOR/ } \\
\text { bolsas y } \\
\text { contenedores }\end{array}$ & Selectiva & $\begin{array}{l}\text { Digestión } \\
\text { anaerobia }\end{array}$ & $\begin{array}{l}\text { - Biogás/energía } \\
\text { eléctrica y calor } \\
\text { - Digestato/sustrato } \\
\text { para la producción de } \\
\text { biomasa algal }\end{array}$ & Liubliana $^{\mathrm{a}, \mathrm{b}}$ & ${ }^{\mathrm{a} O b l a k}$ (2015); ${ }^{\mathrm{b}}$ Kovács (2016) \\
\hline \multirow[t]{2}{*}{11} & $\begin{array}{l}\text { BOR-A/ } \\
\text { bolsas }^{7}\end{array}$ & Selectiva & $\begin{array}{l}\text { Digestión } \\
\text { anaerobia }+ \\
\text { compostaje } \\
\text { del digestato }\end{array}$ & $\begin{array}{l}\text { - Biogás/energía } \\
\text { eléctrica y calor } \\
\text { (usado en distrito de } \\
\text { calefacción }{ }^{8} \text { ) }\end{array}$ & $\begin{array}{l}\text { - Bruselas }{ }^{\mathrm{a}, \mathrm{b}, \mathrm{c}} \\
\text { - Viena }\end{array}$ & $\begin{array}{l}{ }^{a} E C N(2019) ;{ }^{b} P B(2016) ; \\
{ }^{c} \text { BTA International (2016); } \\
{ }^{d} \text { Thon (2013) }\end{array}$ \\
\hline & $\begin{array}{l}\text { BOR-J/bolsas } \\
\text { o contenedores }\end{array}$ & & Compostaje & $\begin{array}{l}\text { - Compost/aplicación } \\
\text { al suelo } \\
\text { Compost/agricultura }\end{array}$ & & \\
\hline 12 & $\begin{array}{l}\text { BOR-A/ } \\
\text { bolsas }\end{array}$ & Conjunta & $\begin{array}{l}\text { Digestión } \\
\text { anaerobia }+ \\
\text { compostaje } \\
\text { del digestato }\end{array}$ & $\begin{array}{l}\text { - Biogás/ } \\
\text { combustible para } \\
\text { buses locales } \\
\text { - Digestato/ } \\
\text { fertilizante líquido y } \\
\text { sólido }\end{array}$ & Oslo ${ }^{\mathrm{a}, \mathrm{b}, \mathrm{c}, \mathrm{d}}$ & $\begin{array}{l}{ }^{\mathrm{a} H a f e e z}(2013) ;{ }^{b} \text { EWTEA } \\
(2013) ;{ }^{c} \text { Wärtsilä (2014); } \\
{ }^{\mathrm{d}} \text { Holmerz (2015) }\end{array}$ \\
\hline
\end{tabular}

Tecnologías identificadas (GCU, capacidad instalada, $\mathrm{t} / \mathrm{d})$

SF: separación en la fuente, GCU: gran centro urbano, BOR: biorresiduos de origen residencial, BOR-A: fracción de residuos de alimentos en los BOR, BOR-J: fracción de residuos de jardín en BOR, ND: no disponible. ${ }^{1}$ Barcelona. ${ }^{2}$ Toronto. ${ }^{3}$ Vancouver. ${ }^{4}$ Berlín. ${ }^{5}$ Múnich. ${ }^{6}$ Zúrich. ${ }^{7}$ Los BOR-A separados voluntariamente son vaciados en contenedores comunitarios (sin bolsa) para su posterior recogida. ${ }^{8}$ Viena. ${ }^{9}$ Suma de capacidades de tratamiento en los digestores anaerobios de biorresiduos separados en la fuente del Ecoparque de Barcelona y de Montcada I Reixac. ${ }^{10}$ Capacidad de la instalación Richmond (no se incluyó la instalación Delta)

(Cuadro VI). Este último se asocia con configuraciones más tecnificadas, ya que sugiere mayores necesidades de infraestructuras para la recolección y el tratamiento de los flujos gestionados, así como de logística.

En cuanto a la presentación de los BOR, contrario a la situación típica de los GCU referentes de ALC sobre el uso de bolsas plásticas, en los sistemastipo de los GCU de AIE la presentación se realiza, principalmente, mediante contenedores retornables $y$, en menor medida, en bolsas biodegradables. Este aspecto se considera importante, ya que el uso de contenedores retornables reduce la presencia de restos de plástico que pudieran alterar la calidad de 
CUADRO VII. CARACTERÍSTICAS DE SISTEMAS DE VALORIZACIÓN DE BIORRESIDUOS ORIGEN RESIDENCIAL EN GRADES CENTROS URBANOS DE PAÍSES DE ALTO INGRESO ECONÓMICO, MEDIANTE OTROS MÉTODOS DE TRATAMIENTO

\begin{tabular}{|c|c|c|c|c|c|c|}
\hline Sistema-tipo & $\mathrm{SF} /$ presentación & $\begin{array}{l}\text { Tipo de } \\
\text { recolección }\end{array}$ & $\begin{array}{l}\text { Método de } \\
\text { tratamiento }\end{array}$ & Producto final/uso & GCU & $\begin{array}{l}\text { Fuentes de } \\
\text { información }\end{array}$ \\
\hline 13 & $\begin{array}{l}\text { BOR-A/bolsas } \\
\text { y contenedores } \\
\text { (incluye contene- } \\
\text { dores inteligentes } \\
\text { comunitarios) }\end{array}$ & Selectiva & $\begin{array}{l}\text { Procesamiento } \\
\text { térmico, } \\
\text { compostaje }^{1}\end{array}$ & $\begin{array}{l}\text { Alimentos húmedos y } \\
\text { secos/alimentos para } \\
\text { animales } \\
\text { - Compost/ } \\
\text { abono para suelos }\end{array}$ & $\begin{array}{l}\text { - Seúl }{ }^{\mathrm{a}} \\
\text { - Daejeon }\end{array}$ & $\begin{array}{l}\text { SMG (2014); } \\
\text { bPadeyanda et al. } \\
(2016)\end{array}$ \\
\hline 14 & $\begin{array}{l}\text { Fracción residual } \\
\text { (incluye BOR)/ } \\
\text { contenedores }\end{array}$ & Conjunta $^{2}$ & $\begin{array}{l}\text { TMB con } \\
\text { digestión } \\
\text { anaerobia }+ \\
\text { compostaje del } \\
\text { digestato }\end{array}$ & $\begin{array}{l}\text { - Biogás/ generación } \\
\text { de energía } \\
\text { - Compost/ } \\
\text { comercializado al } \\
\text { público en general }\end{array}$ & Madrid $^{\mathrm{a}, \mathrm{b}}$ & $\begin{array}{l}{ }^{\mathrm{a}} \mathrm{AM}(2010) ;{ }^{\mathrm{b}} \mathrm{D}- \\
\text { GPTV (2014) }\end{array}$ \\
\hline
\end{tabular}

Tecnologías identificadas

(GCU, capacidad instalada, $\mathrm{t} / \mathrm{d}$ )
Procesamiento térmico (Seúl, 1060), procesamiento térmico (Daejeon, 386), TMB (Madrid, $737^{3}$ )

SF: separación en la fuente, GCU: gran centro urbano, BOR: biorresiduos de origen residencial, BOR-A: fracción de residuos de alimentos en los BOR, TMB: tratamiento mecánico biológico. ${ }^{1}$ Compostaje en menor proporción. ${ }^{2}$ recolección conjunta para biorresiduos. ${ }^{3}$ suma de las capacidades de las plantas de biometanización Las Deshesas y La Paloma

los productos finales para uso en suelos; además, disminuye los problemas operativos y requerimientos de equipos necesarios para separar los biorresiduos de la bolsa plástica en planta.

Por otro lado, en este estudio se ratifica la importancia de la recolección selectiva de los BOR separados en la fuente, en el marco de los sistemas de valorización, realizada en 10 sistemas-tipo, implementados en el $85 \%$ de los GCU referentes de ambos contextos. Asimismo, se resaltan los menores requerimientos de recolección propios de los sistemas de compostaje comunitario implementados en Medellín (Cuadro III) y París (Cuadro V), y la solución de Oslo, que sustitiyó la recolección selectiva usando un sistema de clasificación óptico (Cuadro VI), conservando una gestión diferenciada.

En este estudio se confirmó que el compostaje y la digestión anaerobia son los métodos de tratamiento más utilizados en los GCU referentes alrededor del mundo y en proporción casi igual. No obstante, los métodos de tratamiento utilizados varían en los contextos evaluados. En ALC se evidenció la implementación de compostaje y TMB con compostaje (Cuadro III). En los países de AIE, además del compostaje, se identificó el uso de digestión anaerobia y, en menor proporción, de TMB con digestión anaerobia y producción de alimentos para animales (Cuadro IV).

Las diferencias entre los métodos de tratamiento implementados en los contextos estudiados concuerdan con lo reportado por Thi et al. (2015), quienes reconocieron un mayor uso del compostaje y la poca importancia de la digestión anaerobia en el tratamiento de residuos de alimentos en países en desarrollo. Estas se podrían relacionar, entre otros factores, con los mayores costos de capital y operacionales de la digestión anaerobia (MAAMA 2013), y del TMB basado en ésta, que podrían afectar su viabilidad económica en el contexto de países en desarrollo, tal como fue advertido por CCAP (2012) para Colombia.

Respecto al reciclaje de los BOR-A mediante producción de alimentos para animales, su implementación únicamente se evidenció en GCU referentes asiáticos, estableciendo no sólo una diferencia de contexto entre países de ALC y de AIE, sino también entre las regiones del grupo de países de AIE. Estas diferencias podrían relacionarse con aspectos legales. Por ejemplo, en países asiáticos como la República de Corea, este uso ha sido motivado por leyes locales, debido a la alta demanda del producto obtenido (Thi et al. 2015), y se realiza en condiciones industriales estrictamente controladas que garantizan un consumo seguro de los alimentos producidos (UNEP-ISWA 2015). En países de la Unión Europea se ha prohibido este uso por el temor a infectar a los animales (UNEP-ISWA 2015). En el caso de Estados Unidos se controla el manejo y el tipo de residuos utilizados para este fin; entre ellos, de los residuos que contienen carne (EPA 2016).

Por otro lado, los resultados de este estudio muestran una correspondencia entre los esquemas de separación en la fuente y los métodos de tratamiento 
implementados en los sistemas en funcionamiento. El conjunto de los BOR es tratado tanto mediante compostaje como por digestión anaerobia, mientras que los flujos independientes de BOR-A son tratados, principalmente, mediante digestión anaerobia y producción de alimento para animales, y los de BOR-J mediante compostaje. Por su parte, los flujos de BOR no separados en la fuente son tratados por medio de TMB.

En cuanto a los productos finales y su uso, se evidenció, por un lado, que los sistemas implementados en ALC y AIE que consideran el compostaje, bien sea como tratamiento principal o como postratamiento de la digestión anaerobia (en el caso de AIE), producen compost -utilizado en la jardinería (pública y privada) y en la agricultura (Cuadro V)A partir de las fuentes consultadas (Cuadros V, VI y VII), se confirmó que la comercialización del compost se realiza en algunos GCU tales como Ottawa, San Francisco, Vancouver y Madrid, aspecto importante para la sostenibilidad financiera de las instalaciones de compostaje (UN 2010). Por otro lado, en los sistemas en funcionamiento en AIE se evidenció además la obtención de energía eléctrica, calor, combustible vehicular y biofertilizantes líquidos, como una ventaja comparativa de la digestión anaerobia, los cuales suplen necesidades energéticas de comunidades, instalaciones de tratamiento, vehículos de recolección de residuos y transporte público (Cuadro VI). Como se mencionó, en los sistemas de AIE también se producen alimentos para animales (Cuadro VII).

Por último, al igual que en ALC, pero en menor proporción, también se evidenciaron desafíos de cobertura en sistemas implementados en GCU de AIE como Londres, donde el compostaje de BOR sólo se realiza a los residuos generados en el $9 \%$ de las viviendas (ML 2011); Dublín, donde el $53 \%$ de las residencias cuentan con recolección selectiva de BOR (BiPro-CRI 2015); Viena, donde se trata aproximadamente el $41 \%$ de los biorresiduos generados (BiPro/ CRI 2015), u Oslo, donde se recoge selectivamente el $40 \%$ de los BOR-A (Holmerz 2015).

En la Unión Europea los avances en la gestión de biorresiduos se han atribuido a la implementación de instrumentos como separación en la fuente obligatoria, prohibición de la disposición final de biorresiduos e implementación del esquema de "paga por lo que tires" (PAYT, por sus siglas en inglés) y de impuestos a la disposición final (EEA 2016). Éstos han sido motivados por políticas regionales emitidas (EC 2016) que, inicialmente, se orientaron a la restricción y, por consiguiente, al desvío de los biorresiduos de la disposición final (CE 1999). Posteriormente se enfocaron a la recuperación de recursos mediante valorización (CE 2008, 2015a, b), así como a la consideración de los principios de la economía circular (CE 2015c).

Por último, los GCU de países asiáticos de AIE reportan las mayores coberturas de valorización de residuos de alimentos: 95-97 \% en Seúl (Chrobog 2015) y $99 \%$ en Daejeon (Padeyanda et al. 2016); cifras alcanzadas luego de la prohibición de la disposición final de residuos de alimentos vigente en Corea desde 2005 (LCS 2013, Padeyanda et al. 2016) y de los resultados satisfactorios obtenidos a partir de la implementación de la tecnología de contenedores inteligentes. Lo anterior ha incentivado no sólo una mayor captura de BOR-A por parte del sistema, sino también la reducción de la generación de residuos de alimentos (Chrobog 2015, Padeyanda et al. 2016).

\section{CONCLUSIONES}

La alta proporción de los BOR — y en general de los biorresiduos - en el flujo de RSM en los GCU alrededor del mundo y los impactos ambientales relacionados con su gestión han generado la necesidad de establecer estrategias para su valorización. No obstante, su implementación es mayor en países de AIE, donde se confirmó que al menos 24 GCU valorizan los $\mathrm{BOR}$, primando la aplicación de métodos de tratamiento biológico como compostaje y digestión anaerobia, generalmente precedidos por la separación en la fuente y la recolección selectiva. Por su parte, en la mayoría de los GCU de ALC, la disposición final es la alternativa de gestión de los BOR. En esta región se identificaron tres experiencias de valorización de esta fracción (Ciudad de México, Medellín y Rosario), las cuales difieren en el grado de implementación y en la aplicación de la separación en la fuente y de la recolección selectiva, aunque las tres se enfocan al compostaje.

Aspectos como la reducción del volumen de residuos, la posibilidad de obtener mejoradores de suelos, las prácticas ancestrales milenarias y los menores costos asociados con el compostaje, hacen de este un método de tratamiento atractivo para la valorización de biorresiduos. Adicionalmente se ha impulsado la digestión anaerobia por otros determinantes técnicos y ambientales, frente a situaciones coyunturales que afectan a muchos GCU, tales como déficits energéticos y la necesidad de adoptar enfoques sostenibles que permitan la transición a energías renovables. 
Por su parte, en ALC, la baja implementación de la separación en la fuente y la recolección selectiva de los BOR, en conjunto con la falta de experiencia de la mayoría de los GCU en la ejecución de sistemas que los valoricen, constituyen barreras técnicas y sociales para incrementar el desarrollo de la valorización de esta fracción en la región. No obstante, experiencias como las de Medellín y Ciudad de México sugieren la factibilidad de una gestión diferenciada de esta fracción. En el caso de Ciudad de México, se resalta la factibilidad de construir y operar plantas de compostaje de gran capacidad en la región. Por lo tanto, los sistemas implementados en los GCU referentes pueden constituir puntos de partida para ALC.

Igualmente, considerando la experiencia que ha tenido Ciudad de México con la aplicación de la norma que regula la separación en la fuente y la recolección selectiva de biorresiduos -en tanto que ha contribuido a la producción de compost de buena calidad-, se debe otorgar especial atención a este instrumento en ALC, así como a otros que se han implementado en los países de AIE, en el marco de políticas rectoras a niveles regional y nacional -como el esquema PAYT y la prohibición y aplicación de impuestos a la disposición final de biorresiduos en rellenos sanitarios-.

Por último, se destaca la pertinencia de mejorar la calidad de la información reportada sobre la gestión de residuos sólidos en todos los contextos, en mayor medida en ALC. En este caso, es conveniente la definición y adopción de indicadores que permitan un seguimiento y evaluación comparativa, como han propuesto Wilson et al. (2015), dando respuesta a las necesidades de una gestión integrada circular que precisa información confiable de flujos de fracciones de materiales (es el caso de los BOR). Las necesidades identificadas en el contexto de GCU de ALC reafirman la importancia de la creación de un organismo internacional responsable de la consolidación de información confiable, tal como sugirieron Hernández-Berriel et al. (2016).

\section{LISTA DE ABREVIATURAS}

\begin{tabular}{ll}
\hline AIE & Alto ingreso económico \\
\hline ALC & América Latina y el Caribe \\
\hline BOR & Biorresiduos de origen residencial \\
\hline BOR-A & $\begin{array}{l}\text { Fracción de residuos de alimentos en bio- } \\
\text { rresiduos de origen residencial }\end{array}$ \\
\hline BOR-J & $\begin{array}{l}\text { Fracción de residuos de jardín en biorresi- } \\
\text { duos de origen residencial }\end{array}$ \\
\hline
\end{tabular}

\begin{tabular}{ll}
\hline GCU & Grandes centros urbanos \\
\hline PAYT & Pay-as-you-throw \\
\hline RSM & Residuos sólidos municipales \\
\hline TMB & Tratamiento mecánico biológico \\
\hline t/d & Toneladas/día \\
\hline
\end{tabular}

\section{REFERENCIAS}

AB (2015). Plan de gestión integral de residuos sólidos PGIRS 2016-2027. Alcaldía de Barranquilla-Secretaría de Planeación, Oficina de Desarrollo Económico y Social y Oficina de Hábitat, Barranquilla, Colombia, $178 \mathrm{pp}$.

AB (2016). Recogida de residuos domiciliarios. Ajuntament de Barcelona [en línea]. http://ajuntament. barcelona.cat/ecologiaurbana/es/servicios/la-ciudadfunciona/mantenimiento-del-espacio-publico/gestionde-limpieza-y-residuos/recogida-de-residuos-domiciliarios 11/08/2016

Abarca L., Maas G. y Hogland W. (2013). Solid waste management challenges for cities in developing countries. Waste. Manage. 33 (1), 220-232. https:// doi.org/10.1016/j.wasman.2012.09.008

Acodal-AMVA (2013). Manual de compostaje: manual de aprovechamiento de residuos orgánicos a través de sistemas de compostaje y lombricultura en el Valle de Aburrá. Asociación Colombiana de Ingeniería Sanitaria y Ambiental-Área Metropolitana del Valle de Aburrá. Manual. Medellín, Colombia, 88 pp.

ADRMBH (2015). Plano metropolitano de gestão integrada de resíduos como foco em resíduos de serviços de saúde (RSS) e resíduos da construção civil e volumosos (RCCV). Agência de Desenvolvimento da Região Metropolitana de Belo Horizonte-Goberno de Minas Gerais. Plan. Belo Horizonte, Brasil, 131 pp.

AEB Amsterdam (2018). For a clean society. AEB Amsterdam [en línea]. http://www.aebamsterdam.com/ 19/07/2016

AEM (2018). Tercer informe de gobierno 2009-2012. Indalecio Ríos Velázquez. Ayuntamiento de Ecatepec de Morelos. Informe. Morelos, México, 296 pp.

AM (2010). Guía de buenas prácticas para reducir los residuos urbanos: educación para el consumo sostenible. 2 da ed. Ayuntamiento de Madrid. Guía. Madrid, España, $120 \mathrm{pp}$.

AMC (2012). Avances del plan estratégico Caracas Metropolitana 2020. Alcaldía Metropolitana de Caracas. Informe. Caracas, Venezuela, $238 \mathrm{pp}$.

AMSA S.p.A. (2015). Milán es mi futuro, por eso lo mantengo limpio. Anonima Materie Sintetiche e Affini. Folleto. Milán, Italia, 22 pp. 
AMVA (2014). Experiencias exitosas en el aprovechamiento de residuos en el Valle de Aburrá. Área Metropolitana del Valle de Aburrá. Seminario Internacional Gestión de Residuos Sólidos y Peligrosos-Exporesiduos. Medellín, Antioquia. 19 al 22 de octubre, 2014.

AWM (2012). Renewable energy for Munich - green electricity from biowaste: the AWM dry fermentation plant. Abfallwirtschaftsbetriebe Münster - Der zertifizierte abfallententsorger der Stadt München. Reporte. Múnich, Alemania, 12 pp.

AWM (2016). Das Münchner 3-tonnen-system. Abfallwirtschaftsbetriebe Münster [en línea]. https://www. awm-muenchen.de/privathaushalte/restmuell-papierund-bio/das-3-tonnen-system.html 09/10/2016

BID (2015). Situación de la gestión de residuos sólidos en América Latina y el Caribe. Banco Interamericano de Desarrollo. Folleto recopilatorio. Washington, D. C., EUA, 3 pp.

BiPro-CRI (2015). Assessment of separate collection schemes in the 28 capitals of the EU. BiPro-Copenagen Resource Institute. Reporte final. Bruselas, Bélgica, $161 \mathrm{pp}$.

BSDUDE (2013). Municipal waste management in Berlin. 1ra ed. Berlin Senate Department for Urban Development and the Environment. Folleto. Berlín, Alemania, $51 \mathrm{pp}$.

BSR (2016). Bio-abfall ist biogut: organische abfälle sind im biogut tonne. Bitte wählen Sie einen Bereich [en línea]. http://www.bsr.de/bio_abfaelle.php 10/05/2016

BTA International (2016). References for waste treatment and AD plants. BTA International [en línea]. http://www.bta-international.de/en/referenzen.html $16 / 05 / 2016$

BZ (2016). Bioabfall intelligent nutzen. Biogas Zürich [en línea]. http://www.biogaszuerich.ch/de/bioabfall/ index.php 21/05/2016

C40 Cities (2013). NYC implements comprehensive programs to reduce solid waste. C40 Cities [en línea]. http://www.c40.org/blog_posts/nyc-implementscomprehensive-programs-to-reduce-solid-waste $13 / 05 / 2016$

CA (2019). Organic waste. City of Adelaide [en línea]. https://www.cityofadelaide.com.au/resident/ home-management/recycling-waste/organic-waste/ $15 / 12 / 2019$

Calva-Alejo C.L. y Rojas-Caldelas R.I. (2014). Diagnóstico de la gestión de residuos sólidos urbanos en el municipio de Mexicali, México: retos para el logro de una planeación sustentable. Inf. Tecnol. 25 (3), 59-72. https://doi.org/10.4067/S0718-07642014000300009

Castillo M. (2012). Consultoría para la realización de un estudio de caracterización de residuos sólidos urbanos domésticos y asimilables a domésticos para el Distrito
Metropolitano de Quito. Empresa Pública Metropolitana de Aseo y Secretaría de Ambiente. Informe ejecutivo. Quito, Ecuador, 29 pp.

CAT 23 cities-SCICWM (2014). Tokyo model: The history and strengths of improving municipal waste management operations. Clean Authority of Tokyo 23cities-Study Council on International Cooperation for Waste Management [en línea]. https://www.scribd. com/document/287851491/Tokyo-Model-23-Cities-E $22 / 09 / 2016$

CC (2014). Resource and waste management plan 2018, City of Copenhagen. The technical and environmental administration city development, sustainability. City of Copenhagen. Plan. Copenhague, Dinamarca, 41 pp.

CCAP (2012). Evaluación de NAMA en el sector de residuos en Colombia. Center for Clean Air Policy. Informe. Washington D.C., EUA, 174 pp.

CE (1999). Directiva 1999/31/CE del Consejo de 26 de abril de 1999 relativa al vertido de residuos. Comisión Europea. Directiva. Ciudad de Luxemburgo, Luxemburgo, 19 pp.

CE (2008). Directiva 2008/98/CE del Parlamento Europeo y del Consejo de 19 de noviembre de 2008 sobre los residuos y por la que se derogan determinadas directivas (texto pertinente a efectos del EEE). Comisión Europea. Directiva. Ciudad de Luxemburgo, Luxemburgo, $30 \mathrm{pp}$.

CE (2015a). Propuesta de directiva del Parlamento Europeo y del Consejo, por la que se modifica la directiva 2008/98/CE, sobre los residuos. Comisión Europea. Diario Oficial de la Unión Europea. Propuesta. Bruselas, Bélgica, 28 pp.

CE (2015b). Propuesta de directiva del Parlamento Europeo y del Consejo por la que se modifica la directiva 1999/31/CE, relativa al vertido de residuos (texto pertinente a efectos del EEE). Comisión Europea. Propuesta. Bruselas, Bélgica, 15 pp.

CE (2015c). Comunicación de la Comisión al Parlamento Europeo, al Consejo, al Comité Económico y Social Europeo y al Comité de las Regiones. Comisión Europea. Comunicado. Bruselas, Bélgica, 24 pp.

CESOP (2012). Reporte CESOP núm. 51: residuos sólidos en México. Centro de Estudios Sociales y de Opinión Pública/LXI Legislatura Cámara de Diputados. Reporte. México D. F., México, 52 pp.

Chang N. y Pires A. (2015). Sustainable solid waste management: a systems engineering approach. John Wiley and Sons, Hoboken, Nueva Jersey, EUA, 936 pp. https://doi.org/10.1002/9781119035848

Christensen T.H., Scharff H. y Hjelmar O. (2011). Landfilling: concepts and challenges. En: Solid waste technology and management (T. Christensen, Ed.). John Wiley and Sons, Ltd, Lyngby, Dinamarca, pp. 683-694. 
Chrobog K. (2015). In South Korea, an innovative push to cut back on food waste [en línea]. http://e360.yale. edu/feature/in_south_korea_an_innovative_push_to_ cut_back_on_food_waste $/ 2875 / 10 / 08 / 2016$

CL (2014). Waste strategy 2013-2020: Planning a sustainable future for the City of London. City of London. Plan estratégico. Londres, Inglaterra, $89 \mathrm{pp}$.

CL (2016). Where does your waste and recycling go? City of London [en línea]. http://www.cityoflondon. gov.uk/services/environment-and-planning/wasteand-recycling/household-waste-and-recycling/Pages/ Where-does-your-waste-go.aspx 09/09/2016

CM (2016). Fracció orgànica de recollida municipal. Consell de Mallorca [en línea]. https://www.conselldemallorca.cat/info-pub-ut?\&id_parent=19162\&id class $=13170 \&$ id_section $=12870 \&$ id_son $=1287 \overline{3}$ $10 / 11 / 2016$

CO (2016). Green bin and leaf and yard waste. City of Ottawa [en línea]. https://ottawa.ca/en/garbage-andrecycling/green-bin-and-leaf-and-yard-waste\#whatgoes-your-green-bin 08/08/2016

Cobo S., Domínguez-Ramos A. y Irabien A. (2018). From linear to circular integrated waste management systems: a review of methodological approaches. Resour. Conserv. Recy. 135, 279-295. https://doi.org/10.1016/j. resconrec.2017.08.003

Coffey M. y Coad A. (2010). Collection of municipal solid waste in developing countries. United Nations Human Settlements Programme-UN-HABITAT. Reporte. Nairobi, Kenia, 196 pp.

COGERSA-GPA (2014). Informe anual 2014. Memoria anual de gestión y sostenibilidad del Consorcio para la Gestión de Residuos Sólidos de Asturias y su Sociedad Instrumental COGERSA SAU. Consorcio para la Gestión de Residuos Sólidos de Asturias-Gobierno del Principado de Asturias. Informe. Principado de Asturias, España, 91 pp.

Colef (2010). Diagnóstico de los residuos sólidos urbanos en Tijuana, Baja California. El Colegio de la Frontera Norte. Reporte. Tijuana, México, 88 pp.

Cré (2018). Cré Recommended list of acceptable materials for a household brown bin scheme. Composting and Anaerobic Digestion Association of Ireland. Guía. Enfield, Irlanda, $1 \mathrm{pp}$.

CS (2011). Interim waste strategy: managing the city of Sydney's resources for a sustainable future. City of Sydney. Plan. Sidney, Australia, 111 pp.

CS (2017). Green waste. City of Sydney [en línea]. https://www.cityofsydney.nsw.gov.au/live/waste-andrecycling/green-waste 16/08/2017

CSA (2015). Services guide FY2016. City of San Antonio Solid Waste Management Department. City of San Antonio. Guía. San Antonio, Texas, EUA, 26 pp.
CT (2016). Green bin (organics). City of Toronto [en línea]. https://www.toronto.ca/services-payments/ recycling-organics-garbage/houses/what-goes-in-mygreen-bin/ 16/05/2016

CV (2016). Recycling guidelines and service. City of Vancouver [en línea]. http://vancouver.ca/home-propertydevelopment/recycling.aspx 18/06/2016

DAPM-Univalle (2006). Caracterización de los residuos sólidos residenciales generados en el municipio de Santiago de Cali - 2006. Departamento Administrativo de Planeación Municipal-Universidad del Valle. Informe. Santiago de Cali, Colombia, 26 pp.

DAPM (2015). Plan de gestión integral de residuos sólidos - PGIRS 2015-2027. Departamento Administrativo de Planeación Municipal-Alcaldía de Santiago de Cali. Plan. Cali, Colombia, 733 pp.

DGPTV (2014). Memoria de actividades del Parque Tecnológico Valdemingómez. Dirección General del Parque Tecnológico de Valdemingómez. Memoria. Madrid, España, 134 pp.

Dickenson I. (2013). Anaerobic organics processing, Toronto's new disco road green bin processing facility. Solid Waste and Recycling 18 (3), 16-21.

Drescher S. y Zurbrügg C. (2006). Decentralised composting: lessons learned and future potentials for meeting the millennium development goals. Paper. Solid waste, health and the millennium development goals. CWGWASH Workshop. Kolkata, India. 1-5 de febrero, 2006. 1-9 pp.

EB (2009). Metanización y cogeneración. Ecoparc de Barcelona [en línea]. http://ecoparcben.com/contenido. php?id=79 11/08/2016

EC (2016). Background report on best environmental management practice in the waste management sector. European Commission. Reporte. Bruselas, Bélgica, 399 pp.

ECN (2019). ECN country report 2018 Belgium (Flanders). European Compost Network. Reporte. Flandes, Bélgica, $11 \mathrm{pp}$.

EEA (2013). Managing municipal solid waste - a review of achievement in 32 Europeans countries. European Environmental Agency, European Union. Reporte. Ciudad de Luxemburgo, Luxemburgo, 36 pp. https:// doi.org/10.2800/71424

EEA (2016). Municipal waste management across European countries. European Environmental Agency, European Union. Reporte. Copenhague, Dinamarca, 7 pp. https://doi.org/10.2800/475915

EMR (2016). Ecoparc de Montcada i Reixac. Ecoparc de Montcada i Reixac [en línea]. http://www3.amb.cat/ ema/visites/eco2/adults/ 11/08/2016

EPA (2016). Composting and anaerobic digestion in Ireland. Environmental Protection Agency. Informe ejecutivo. Wexford, Irlanda, 1 pp. 
EWTEA (2013). Biogas and biofertilizer. Ege Waste-ToEnergy Agency, City of Oslo. Folleto. Oslo, Noruega, $2 \mathrm{pp}$.

Ferreras R. (2015). Los desechos sólidos en la República Dominicana: su proceso y destino final. Observatorio Político Dominicano - Unidad de Gobierno Local. Informe. Santo Domingo, República Dominicana, $10 \mathrm{pp}$.

FIUBA-CEAMSE (2011). Estudio de la calidad de los residuos sólidos urbanos de Área Metropolitana de Buenos Aires. Tercer informe de avance. Facultad de Ingeniería de la Universidad de Buenos Aires - Coordinación Ecológica Área Metropolitana Sociedad del Estado. Informe. Buenos Aires, Argentina, 159 pp.

Gándara M. y Guerrero E. (2013). Indicadores ambientales para la gestión de los residuos sólidos domiciliarios en Rosario, Argentina. DELOS, Desarrollo Local Sostenible 6 (16), 1-18.

GB (2018). Plano distrital de gestão integrada de resíduos sólidos - PDGIRS. Governo de Brasília - Secretaria de Estado de Infraestrutura e Serviços Públicos do Distrito Federal. Plan. Brasília, Brasil, 409 pp.

GEJ (2017). Programa estatal para la prevención y gestión integral de residuos del Estado de Jalisco. Gobierno del Estado de Jalisco - Secretaría de Medio Ambiente y Desarrollo Territorial. Programa. Jalisco, México, $387 \mathrm{pp}$.

GEP (2011). Plano metropolitano de resíduos sólidos - PMRS. Região Metropolitana do Recife - RMR. Governo do Estado de Pernambuco - Secretaria Das Cidades. Plan. Recife, Brasil, 85 pp.

Gorrie P. (2015). Toronto expands anaerobic digestion of source separated organics. Biocycle 56 (2), 40.

Hafeez M.S. (2013). Comparison of solid waste management between Oslo (Norway) and Lahore (Pakistan). Tesis de Maestría. Department of Noragric, Norwegian University of Life Sciences. Ås, Noruega, 51 pp.

Hagney M. (2016). Watch: city expands composting services to all residents [en línea]. https://therivardreport. com/watch-city-expands-composting-services-tomore-residents/ 08/08/2016

Hamatschek E. y Faulstich M. (2010). Municipal solid waste management in Chile: current system and energy potential of MSW. J. Solid Waste Technol. Manag. 36 (1), 594-604.

Hernández-Berriel M., Aguilar-Virgen Q., TaboadaGonzález P., Lima-Morra R., Eljaiek-Urzola M., Márquez-Benavides L. y Buenrostro-Delgado O. (2016). Generación y composición de los residuos sólidos urbanos en América Latina y el Caribe. Rev. Int. Contam. Ambie. 32, 11-22. https://doi.org/10.20937/ RICA.2016.32.05.02

Holmerz S. (2015). Oslo's colourful solution to waste management [en línea]. https://waste-management-world. com/a/oslo-s-colourful-solution-to-waste-management $22 / 05 / 2016$

Hoornweg D. y Bhada-Tata P. (2012). What a waste: a global review of solid waste management. The World Bank. Reporte. Washington D. C., EUA, 116 pp.

IBAM (2010). Plano diretor de resíduos sólidos de Manaus. Instituto Brasileiro de Administração - Municipal Prefeitura Municipal de Manaus - Programa Social e Ambiental dos Igarapés de Manaus - Unidade de Gerenciamento do Programa Social e Ambiental dos Igarapés de Manaus - Banco Interamericano de Desarrollo - Amazonas Governo do Estado. Plan. Manaus, Brasil, 154 pp.

ICONTEC (2011). Norma Técnica Colombiana NTC 5167 - 2011-03-23. Productos para la industria agrícola. Productos orgánicos usados como abonos o fertilizantes y enmiendas o acondicionadores de suelos. Instituto Colombiano de Normas Técnicas y Certificación. Bogotá, Colombia, 23 de marzo de 2011.

IDEAM (2017). Acciones de mitigación del cambio climático en Colombia. Tercera Comunicación Nacional de Cambio Climático. Instituto de Hidrología, Meteorología y Estudios Ambientales - Programa de las Naciones Unidas para el Desarrollo - Ministerio de Ambiente y Desarrollo Sostenible - Departamento Nacional de Planeación - Cancillería - Fondo para el Medio Ambiente Mundial. Informe. Bogotá D. C., Colombia, 128 pp.

Ineco-Tragsatec (2016). Plan maestro de gestión integral de residuos del Distrito Metropolitano de Quito. Ineco - Corporación Andina de Fomento - Secretaría de Ambiente - Quito Alcaldía. Plan. Quito, Ecuador, 287 pp.

IPEA (2012). Diagnóstico dos resíduos sólidos urbanos. Relatório de pesquisa. Instituto de Pesquisa Económica Aplicada, Governo Federal. Informe. Brasília, Brasil, 82 pp.

Jacobi P.R. y Besen G.R. (2011). Gestão de resíduos sólidos em São Paulo: desafios da sustentabilidade. Estud. Avançados 25 (71), 135-158. https://doi.org/10.1590/ S0103-40142011000100010

JRC-IES (2011). Supporting environmentally sound decisions for bio-waste management: a practical guide to Life Cycle Thinking (LCT) and Life Cycle Assessment (LCA). Joint Research Centre, European Commission - Institute for Environment and Sustainability. Guía. Ispra, Italia, 94 pp. https://doi.org/10.2788/53942

Kawai K. y Tasaki T. (2016). Revisiting estimates of municipal solid waste generation per capita and their reliability. J. Mater. Cycles Waste 18 (1), 1-13. https:// doi.org/10.1007/s10163-015-0355-1

Kaza S., Yao L., Bhada-Tata P. y Van Woerden F. (2018). What a waste 2.0: a global snapshot of solid waste management to 2050. Urban development. World Bank, Washington D. C., EUA, 295 pp. 
KK (2014). Regulativ for husholdningsaffald. København Kommune. Reglamentos. Copenhague, Dinamarca, 38 pp.

Kovács E. (2016). Success stories: anaerobic digestion of biodegradable municipal solid waste in European cities. European Biogas Association. Folleto. Bruselas, Bélgica, 8 pp.

Kumar A. y Samadder S.R. (2017). A review on technological options of waste to energy for effective management of municipal solid waste. Waste Manage. 69, 407422. https://doi.org/10.1016/j.wasman.2017.08.046

LCS (2013). Information note: South Korea's waste management policies. Research Office. Legislative Council Secretariat. Nota informativa. Seúl, Corea del Sur, 24 pp.

Levis J.W., Barlaz M.A., Themelis N.J. y Ulloa P. (2011). Assessment of the state of food waste treatment in the United States and Canada. Waste Manage. 30 (8-9), 1486-1494. http://doi.org/10.1016/j.wasman.2010.01.031

MAAMA (2013). Gestión de biorresiduos de competencia municipal: guía para la implantación de la recogida separada y tratamiento de la fracción orgánica. Ministerio de Agricultura, Alimentación y Medio Ambiente de España. Guía. Madrid, España, 342 pp.

Manfredi S., Cristóbal J., Torres de Matos C., Giavini M., Vasta A., Sala S., Saouter E. y Tuomisto H. (2015). Improving sustainability and circularity of European food waste management with a life cycle approach. Joint Research Centre, European Union. Reporte European Commission. Reporte. Bruselas, Bélgica, 79 pp. https://doi.org/10.2788/182997

Mansur G. (2015). Tratamento mecânico biológico para valorização de RSU: caso de sucesso em Brasília/D. F. Memorias. Decimo Primeiro Encontro Técnico de Alto Nivel Compostagem. São Paulo, Brasil. 01 al 02 de octubre de 2015. PDF.

Minam Perú (2012). Cuarto informe nacional de residuos sólidos municipales y no municipales: gestión 20102011. Ministerio del Ambiente Perú - Viceministerio de Gestión Ambiental de Perú. Informe. Lima, Perú, 379 pp.

ML (2011). London's wasted resource the mayor's municipal waste management strategy. Greater London Authority. Estrategia. Londres, Inglaterra, 135 pp.

MMA-A (2011). Prevención de la generación, aprovechamiento y cadena productiva de los residuos sólidos. Ministerio de Medio Ambiente y Agua de Bolivia. Diagnóstico. La Paz, Bolivia, 40 pp.

Montello S.p.A. (2015). Processing plant for waste of organic origin. Montello S.p.A. Industria del Recupero e Reciclo [en línea]. http://joomla.montello-spa.it/en/ index.php?option $=$ com_content $\&$ task $=$ view $\&$ id $=22 \&$ Itemid=42 07/06/2016
MP (2017). Plan compost Parisien 2016-2020. Mairie de Paris [en línea]. https://api-site-cdn.paris.fr/images/89698 30/11/2017

MR (2017a). Planta de compostaje Bella Vista. Municipalidad de Rosario [en línea]. https:/www.rosario. gov.ar/web/ciudad/medio-ambiente/reciclado/plantade-compostaje-bella-vista 07/06/2017

MR (2017b). Barrios verdes recolección alterna por tipo de residuos. Municipalidad de Rosario [en línea]. https:// www.rosario.gov.ar/web/servicios/higiene-urbana/ recoleccion-de-residuos/barrios-verdes-recoleccionalterna-por-tipo-de 07/06/2017

MSCR (2016). Plan nacional de gestión integral de residuos 2016-2021. Ministerio de Salud de Costa Rica - Gobierno de la República de Costa Rica. Plan. San José, Costa Rica, 116 pp.

Neto P.N. y Moreira T.A. (2009). Gestão de resíduos sólidos urbanos na região metropolitana de Curitiba: política regional de compostagem. Revista Geografar. 4 (2), 72-96.

Noguera K.M. y Olivero J. (2010). Los rellenos sanitarios en Latinoamérica: caso colombiano. Revista Acad. Colom. Ci. Exact. 34 (132), 347-356.

NSWEPA (2014). NSW waste avoidance and resource recovery strategy 2014-21. New South Wales Environment Protection Authority. Reporte. Sidney, Australia, 37 pp.

Oblak E. (2015). Case study \# 5: the story of Ljubljana. Zero waste Europe. Caso de estudio. Liubliana, Eslovenia, $6 \mathrm{pp}$.

Ojeda-Benítez S., Armijo de Vega C. y Ramí M.E. (2003). Characterization and quantification of household solid wastes in a Mexican city. Resour. Conserv. Recycl. 39 (3), 211-222. https://doi.org/10.1016/S09213449(03)00028-4

OPS (2005). Informe de la evaluación regional de los servicios de manejo de residuos sólidos municipales en América Latina y el Caribe. Organización Panamericana de la Salud. Informe. Washington D.C., EUA, $128 \mathrm{pp}$.

Orgaworld (2014). Tunnel composting Ottawa (Ontario), Canada: tailor-made to deal with (extreme) local conditions [en línea]. http://www.orgaworld.com/whatwe-are-good/taylor-made-plants-and-installations/ tunnel-composting-ottawa-ontario-canada 08/08/2016

Padeyanda Y., Jang Y., Ko Y. y Yi S. (2016). Evaluation of environment impacts of food waste management by material flow analysis (MFA) and life cycle assessment (LCA). J. Mater. Cycles Waste 18 (3), 493-508. https:// doi.org/10.1007/s10163-016-0510-3

Paris (2016). Réduire ses déchets [en línea]. https://www. paris.fr/gaspillage\#composter-ses-biodechets $\% 2011$ $10 / 08 / 2016$ 
PB (2016). Waste processing. Propreté Brussels [en línea]. https://www.arp-gan.be/en/waste-processing. html 25/05/2016

PC (2018). Coordenadoria de tratamento de resíduos. Prefeitura de Campinas [en línea]. http://www. campinas.sp.gov.br/governo/servicos-publicos/dlu/ coordenadoria-tr.php 02/01/2015

PCRJ (2012). Plano municipal de gestão integrada de resíduos sólidos - PMGIRS da Cidade do Rio de Janeiro 2012-2016. Prefeitura da Cidade do Rio de Janeiro. Plan. Río de Janeiro, Brasil, 80 pp.

Penido-Monteiro J.H. (2012). A gestão sustentável de residuos na cidade do Rio de Janeiro. COMLURB - Cia. - Municipal de Limpieza Urbana do Rio de Janeiro. Diapositivas. Río de Janeiro, Brasil, 57 pp.

PF (2012). Plano municipal de gestão integrada de resíduos sólidos de Fortaleza Estado do Ceará. Prefeitura de Fortaleza. Plan. Fortaleza, Brasil, 284 pp.

PG (2011). Plano diretor de resíduos sólidos de Guarulhos. Prefeitura de Guarulhos -Secretaria de Serviços Públicos - Departamento de Limpieza Urbana. Plan. Guarulhos, Brasil, 264 pp.

PMC (2010). Plano de gestão integrada de resíduos sólidos. Prefeitura Municipal de Curitiba - Secretaria Municipal do Meio Ambiente. Plan. Curitiba, Brasil, $121 \mathrm{pp}$.

PPA (2013). Plano municipal de gestão integrada de resíduos sólidos. Volumen 1 - diagnóstico e prognóstico. Prefeitura Municipal de Porto Alegre - Departamento Municipal de Limpieza Urbana. Plan. Porto Alegre, Brasil, 372 pp.

Prudencio C. (2010). Gestão integrada de resíduos sólidos no Rio De Janeiro: impactos das decisões dos gestores nas políticas públicas. Tesis de Doctorado. Programa de Posgrado en Planificación Eléctrica. Universidad Federal de Río de Janeiro. Río de Janeiro, Brasil, 290 pp.

Recology (2016). Jepson Prairie Organics [en línea]. https://www.recology.com/recology-vacaville-solano/ jepson-prairie-organics/ 13/05/2016

Ríos R. y Mendoza R. (2013). Aprovechamiento de los residuos sólidos mediante producción de compost en el Departamento de Santa Cruz. Facultad de Ciencias Exactas y Tecnología de la Universidad Autónoma Gabriel Rene Moreno. Informe. Santa Cruz, Bolivia, $21 \mathrm{pp}$.

RN (2018). Food waste. Recycle now [en línea]. https:// www.recyclenow.com/recycling-knowledge/how-isit-recycled/food-waste 25/05/2018

Rosso M.E. y Marcolini I.C. (2015). Planta de tratamiento mecánico biológico (TMB) - Norte III Ceamse. Provincia de Buenos Aires, Argentina. FuturEnviro, 74-80.

Saveyn H. y Eder P. (2014). End-of-waste criteria for biodegradable waste subjected to biological treatment (compost \& digestate): technical proposals. European Commission - JRC Scientific and Policy Reports. Reporte. Luxemburgo, Luxemburgo, 312 pp.

SEDEMA(2015). Programa de gestión integral de residuos sólidos - PGIRS 2016-2020. Secretaría del Medio Ambiente de la Ciudad de México - Secretaría de Obras y Servicios. Ciudad de México, México, 49 pp.

SEDEMA (2018). Inventario de residuos sólidos CDMX 2017. Secretaría del Medio Ambiente de la Ciudad de México. Informe. Ciudad de México, México, 163 pp. SETIN (2010). Plano municipal de saneamiento básico. Volumen II - Sistemas de abastecimiento de água e esgotamento sanitário de Salvador. Secretaria Municipal dos Transportes Urbanos e Infraestrutura - Prefeitura Municipal do Salvador. Plan. Salvador, Brasil, 218 pp.

SFE (2016). Residential recycling and composting: recycling and composting could help save money. SF Environment [en línea]. https://sfenvironment.org/ zero-waste-in-SF-is-recycling-composting-and-reuse 25/07/2016

SFE (2017). What happens during composting and why do we do it? SF Environment [en línea]. https://sfenvironment.org/solution/what-happens-during-compostingand-why-do-we-do-it 25/07/2016

SGCT-UDEM (2015). Actualización del plan de gestión integral de residuos sólidos (PGIRS) del municipio de Medellín, dando cumplimiento a la resolución 0754 del 25 de noviembre del 2014. Secretaría de Gestión y Control Territorial y Universidad de Medellín. Plan de gestión. Medellín, Colombia, 1024 pp.

Sinoski K. (2012). Metro Vancouver has big plans to convert organic waste to energy or compost [en línea]. http:/www.vancouversun.com/business/Metro+Vanc ouver+plans + convert+organic + waste+energy+comp ost/7310604/story.html 19/06/2016

SLU (2018). Coleta seletiva. Serviço de Limpeza Urbana do Distrito Federal [en línea]. http://www.slu.df.gov. br/mapa-da-coleta-seletiva-em-sua-quadra/ 9/05/2018

SLUM-PM (2018). Coleta domiciliar e limpieza urbana. Superintendência de Limpeza Urbana de Maceió Prefeitura de Maceió [en línea]. http://www.maceio. al.gov.br/slum/coleta-domiciliar-e-limpeza-urbanaslum/0 18/10/2017

SMADF (2012). Norma ambiental para el Distrito Federal NADF-020-AMBT-2011, que establece los requerimientos mínimos para la producción de composta a partir de la fracción orgánica de los residuos sólidos urbanos, agrícolas, pecuarios y forestales, así como las especificaciones mínimas de calidad de la composta producida y/o distribuida en el Distrito Federal. Secretaría del Medio Ambiente del Distrito Federal. Gaceta Oficial del Distrito Federal, 30 de noviembre. 
SMADF (2015). Norma ambiental para el Distrito Federal NADF-024-AMBT-2013, que establece los criterios y especificaciones técnicas bajo los cuales se deberá realizar la separación, clasificación, recolección selectiva y almacenamiento de los residuos del distrito federal. Secretaría del Medio Ambiente del Distrito Federal. Gaceta Oficial del Distrito Federal, 8 de julio.

SMG (2014). Environment and energy news: Seoul announces "zero" landfilling of garbage up to 2017. Seoul Metropolitan Government [en línea]. http:// english.seoul.go.kr/seoul-announces-zero-landfillinggarbage-2017/ 07/09/2016

SO (2015). Athens-biowaste: biowaste separation at the source. Sustainable Greece 2020. Practices of sustainable development, responsible entrepreneurship \& social responsibility. Sustainability Observatory [en línea]. http://observatory.sustainablegreece2020. com/en/practice/athens-biowaste-biowaste-separationsource. 836.html 11/07/2016

SSPD de Colombia-DNP (2017). Disposición final de residuos sólidos. Informe nacional - 2016. Superintendencia de Servicios Públicos Domiciliarios de Colombia y Departamento Nacional de Planeación. Informe. Bogotá D. C., Colombia, 78 pp.

SZ (2016). Bioabfall. Stadt Zürich [en línea]. https://www. stadt-zuerich.ch/content/ted/de/index/entsorgung_recycling/sauberes_zuerich.html 26/05/2016

Tello P., Martínez E., Daza D., Soulier M. y Terraza H. (2010). Informe de la evaluación regional de los servicios de manejo de residuos sólidos municipales en América Latina y el Caribe. Organización Panamericana de la Salud - Asociación Interamericana de Ingeniería Sanitaria y Ambiental - Banco Interamericano de Desarrollo. Informe. Informe. Washington, D. C., EUA, 158 pp.

Themelis N.J. y Díaz-Barriga M.E. (2012). Estudio de prefactibilidad técnica y económica para la instalación de capacidad de generación de energía a partir de residuos (WTE) en Uruguay. Technologies for Sustainable Waste Management-Themelis Associates y Dirección Nacional de Medio Ambiente-Ministerio de Vivienda, Ordenamiento Territorial y Medio Ambiente. Estudio. Montevideo, Uruguay, $122 \mathrm{pp}$.

Thi N., Kumar G. y Lin C. (2015). An overview of food waste management in developing countries: current status and future perspective. J. Environ. Manage. 157, 220-229. https://doi.org/10.1016/j.jenvman.2015.04.022

Thon J. (2013). Biowaste management in Vienna. City of Vienna - Municipal Department 48 - Waste Management, Street Cleaning and Vehicle Fleet. Folleto. Viena, Austria, $12 \mathrm{pp}$.
TIRME (2013). Informe de sostenibilidad 2013. Parque de Tecnologías Ambientales de Mallorca. Informe. Palma de Mallorca, España, 156 pp.

TIRME (2015). Planta de metanización. Parque de Tecnologías Ambientales de Mallorca [en línea]. http:// www.tirme.com/es/metanizacion_02f9s $11 . h t m l$ 20/08/2016

Troschinetz A. y Mihelcic J. (2009). Sustainable recycling of municipal solid waste in developing countries. Waste Manage. 29 (2), 915-923. https://doi. org/10.1016/j.wasman.2008.04.016

UAESP (2011). Caracterización de los residuos sólidos residenciales generados en la ciudad de Bogotá D.C. Unidad Administrativa Especial de Servicios Públicos - Alcaldía Mayor de Bogotá D.C. Hábitat. Bogotá D. C., Colombia, 69 pp.

UAESP (2015). Actualización plan de gestión integral de residuos sólidos 2016-2027 de Bogotá D.C. Hábitat Unidad Administrativa Especial de Servicios Públicos - Alcaldía Mayor de Bogotá D.C. Plan. Bogotá D. C., Colombia, $44 \mathrm{pp}$.

UDEM (2015). Estudio de caracterización de residuos sólidos generados en el sector residencial del municipio de Medellín y sus cinco corregimientos. Universidad de Medellín, Facultad de Ingeniería, Programa de Ingeniería Ambiental - Grupo de Extensión - Alcaldía de Medellín. Informe. Medellín, Colombia, 180 pp.

UN (2014). World economic situation and prospects. United Nations. Reporte. Nueva York, EUA, 181 pp.

UNEP-ISWA (2015). Global waste management outlook. Summary for decision-makers. United Nations Environment Programme - International Solid Waste Association. Reporte. Osaka, Japón - Viena, Austria, 8 pp.

UN-Habitat (2010). Solid waste management in the world's cities: water and sanitation in the world's cities. United Nations Human Settlements Programme. Reporte. Londres, Inglaterra, $228 \mathrm{pp}$.

USEPA (2016). Advancing sustainable materials management: 2014 Fact Sheet. United States Environmental Protection Agency. Reporte. Washington D. C., EUA, $22 \mathrm{pp}$.

VE (2011). City of Ottawa green bin program project completion report: Federation of Canadian Municipalities - Green municipal fund project 9346. Ottawa, Viridis Environmental. Reporte. Ottawa, Canadá, 20 pp.

Vögeli Y., Lohri C.R., Gallardo A., Diener S. y Zurbrügg C. (2014). Anaerobic digestion of biowaste in developing countries: practical information and case studies. Department of Water and Sanitation in Developing Countries - Sandec - Swiss Federal Institute of Aquatic Science and Technology - Eawag. Dübendorf, Suiza, $135 \mathrm{pp}$. 
Wärtsilä (2014). Wärtsilä biogas liquefaction plant EGE biogas, Oslo, Norway. Wärtsilä - Enabling sustainable societies with smart technology. Folleto. Noruega, Oslo, 4 pp.

Wilson D., Rodic L., Scheinberg A., Velis C.A. y Alabaster G. (2012). Comparative analysis of solid waste management in 20 cities. Waste Manage. Res. 30 (3), 237-254. https://doi.org/10.1177/0734242X1 2437569
Wilson D.C., Rodic L., Cowing M.J., Velis C.A., Whiteman A.D., Scheinberg A., Vilches R., Masterson D., Stretz J. y Oelz B. (2015). 'Wasteaware' benchmark indicators for integrated sustainable waste management in cities. Waste Manage. 35, 329-342. https:// doi.org/10.1016/j.wasman.2014.10.006

Zaman A.U. (2014). Measuring waste management performance using the 'zero waste index': the case of Adelaide, Australia. J. Clean. Prod. 66, 407-419. https://doi.org/10.1016/j.jclepro.2013.10.032 